SUPPLEMENTARY MATERIAL

New monoterpenoid alkaloids from the aerial parts of Uncaria hirsuta

Jun-Feng Jia, , Yuan Zhang, Xiao-Jun Huang ${ }^{\mathrm{a}, \mathrm{b}}$, Sheng-Yuan Zhang, Hai-Yan Tian ${ }^{\mathrm{a}}$, Lei Wang ${ }^{\mathrm{a}, \mathrm{b}, *}$, Wen-Cai Ye $\mathrm{e}^{\mathrm{a}, \mathrm{b}, *}$

${ }^{a}$ Institute of Traditional Chinese Medicine \& Natural Products, College of Pharmacy, Jinan University, Guangzhou 510632, P.R. China

${ }^{b}$ JNU-HKUST Joint Laboratory for Neuroscience \& Innovative Drug Research, Jinan University, Guangzhou 510632, P.R. China

*Corresponding author. E-mail: chyewc@gmail.com; cpuwanglei@126.com 


\section{New monoterpenoid alkaloids from the aerial parts of Uncaria}

\section{hirsuta}

To investigate the chemical constituents of medicinal plant Uncaria hirsuta, three new monoterpenoid alkaloids, named hirsutanines A-C (1-3), were isolated. Their structures with absolute configurations were elucidated by means of NMR, X-ray diffraction, and CD analysis. Compound 3 was the first dimeric monoterpenoid alkaloid obtained from genus Uncaria.

Keywords: Rubiaceae; Uncaria hirsuta; alkaloids

\section{Supplementary data for the paper:}

Figure S1 $\quad{ }^{1} \mathrm{H}$ NMR spectrum of compound 1 in DMSO- $d_{6}(300 \mathrm{MHz}) . \quad 4$

Figure S2 ${ }^{13} \mathrm{C}$ spectrum of compound 1 in DMSO-d $(75 \mathrm{MHz})$. 4

Figure S3 DEPT-135 spectrum of compound 1 in DMSO-d $d_{6}(75 \mathrm{MHz}) . \quad 5$

Figure S4 $\quad{ }^{1} \mathrm{H}-{ }^{1} \mathrm{H}$ COSY spectrum of compound $\mathbf{1}$ in DMSO- $d_{6}$. 5

Figure S5 HSQC spectrum of compound $\mathbf{1}$ in DMSO- $d_{6}$. 6

Figure S6 HMBC spectrum of compound 1 in DMSO- $d_{6}$. 6

$\begin{array}{lll}\text { Figure S7 NOESY spectrum of compound } \mathbf{1} \text { in DMSO- } d_{6} \text {. } & 7\end{array}$

$\begin{array}{lll}\text { Figure S8 HR-ESI-MS spectrum of } \mathbf{1} & 7\end{array}$

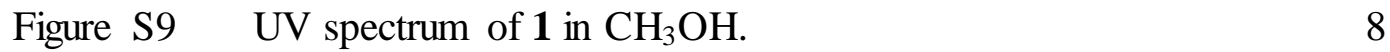

$\begin{array}{lll}\text { Figure S10 } & \text { IR (KBr disc) spectrum of } \mathbf{1} . & 8\end{array}$

$\begin{array}{lll}\text { Figure S11 Single crystal X-ray data and structure of } \mathbf{1} \text {. } & 15\end{array}$

Figure S12 ${ }^{1}$ H NMR spectrum of compound 2 in DMSO- $d_{6}(300 \mathrm{MHz}) . \quad 16$

Figure S13 ${ }^{13} \mathrm{C}$ spectrum of compound 2 in DMSO-d $(75 \mathrm{MHz})$. 16

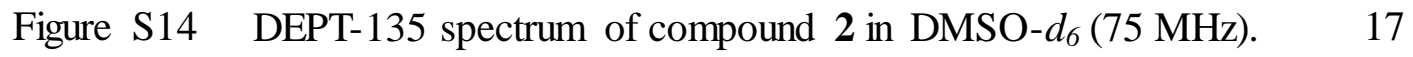

Figure S15 ${ }^{1} \mathrm{H}-{ }^{1} \mathrm{H}$ COSY spectrum of compound 2 in DMSO- $d_{6}$. 17

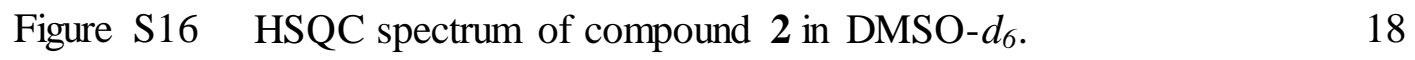


Figure S17 HMBC spectrum of compound 2 in DMSO- $d_{6}$. 18

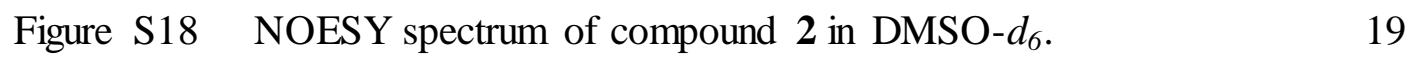

$\begin{array}{lll}\text { Figure S19 HR-ESI-MS spectrum of } 2 . & 19\end{array}$

$\begin{array}{lll}\text { Figure S20 UV spectrum of } 2 \text { in } \mathrm{CH}_{3} \mathrm{OH} \text {. } & 20\end{array}$

$\begin{array}{lll}\text { Figure S21 IR (KBr disc) spectrum of } 2 . & 20\end{array}$

Figure S22 ${ }^{1} \mathrm{H}$ NMR spectrum of compound 3 in DMSO- $d_{6}(300 \mathrm{MHz}) . \quad 21$

Figure S23 ${ }^{13} \mathrm{C}$ spectrum of compound 3 in DMSO- $d_{6}(75 \mathrm{MHz})$.

Figure S24 DEPT-135 spectrum of compound 3 in DMSO-d $d_{6}(75 \mathrm{MHz}) . \quad 22$

Figure S25 ${ }^{1} \mathrm{H}-{ }^{1} \mathrm{H}$ COSY spectrum of compound 3 in DMSO- $d_{6}$. 22

Figure S26 HSQC spectrum of compound 3 in DMSO- $d_{6}$. 23

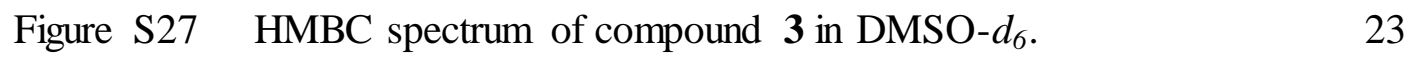

$\begin{array}{lll}\text { Figure S28 NOESY spectrum of compound } 3 \text { in DMSO- } d_{6} \text {. } & 24\end{array}$

Figure S29 HR-ESI-MS (a) and MS/MS (b) of compound 3 (negative 25 mode) and the proposed fragmentation pathway (c).

Figure S30 Key ${ }^{1} \mathrm{H}-{ }^{1} \mathrm{HCOSY}$ and HMBC correlations of $\mathbf{1}$ and 2.

$\begin{array}{lll}\text { Figure S31 Key NOESY correlations of } \mathbf{1} & 26\end{array}$

$\begin{array}{lll}\text { Figure S32 Key HMBC correlations of } 3 . & 26\end{array}$ 


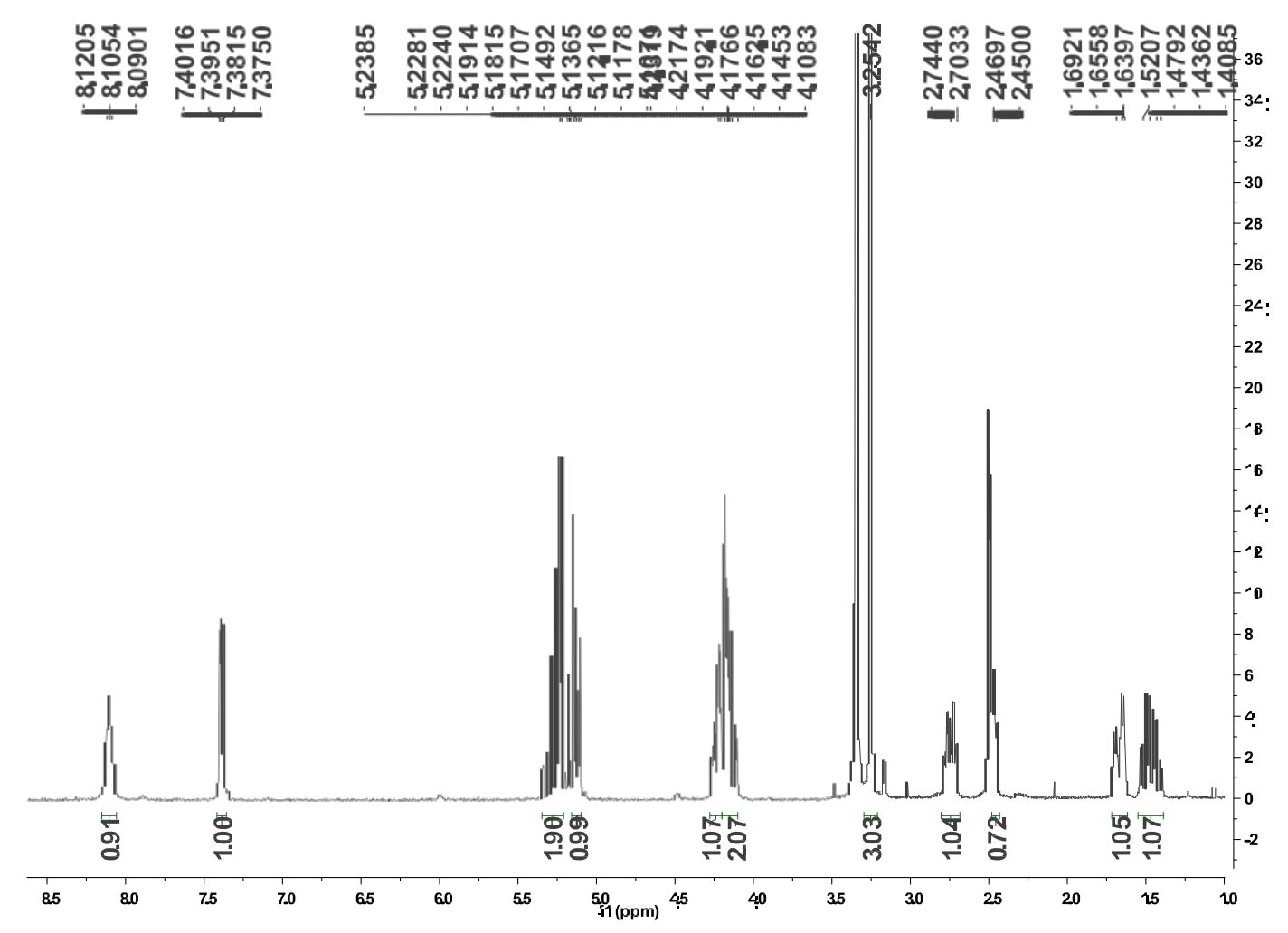

Figure S1. ${ }^{1} \mathrm{H}$ NMR spectrum of compound 1 in DMSO-d $(300 \mathrm{MHz})$.

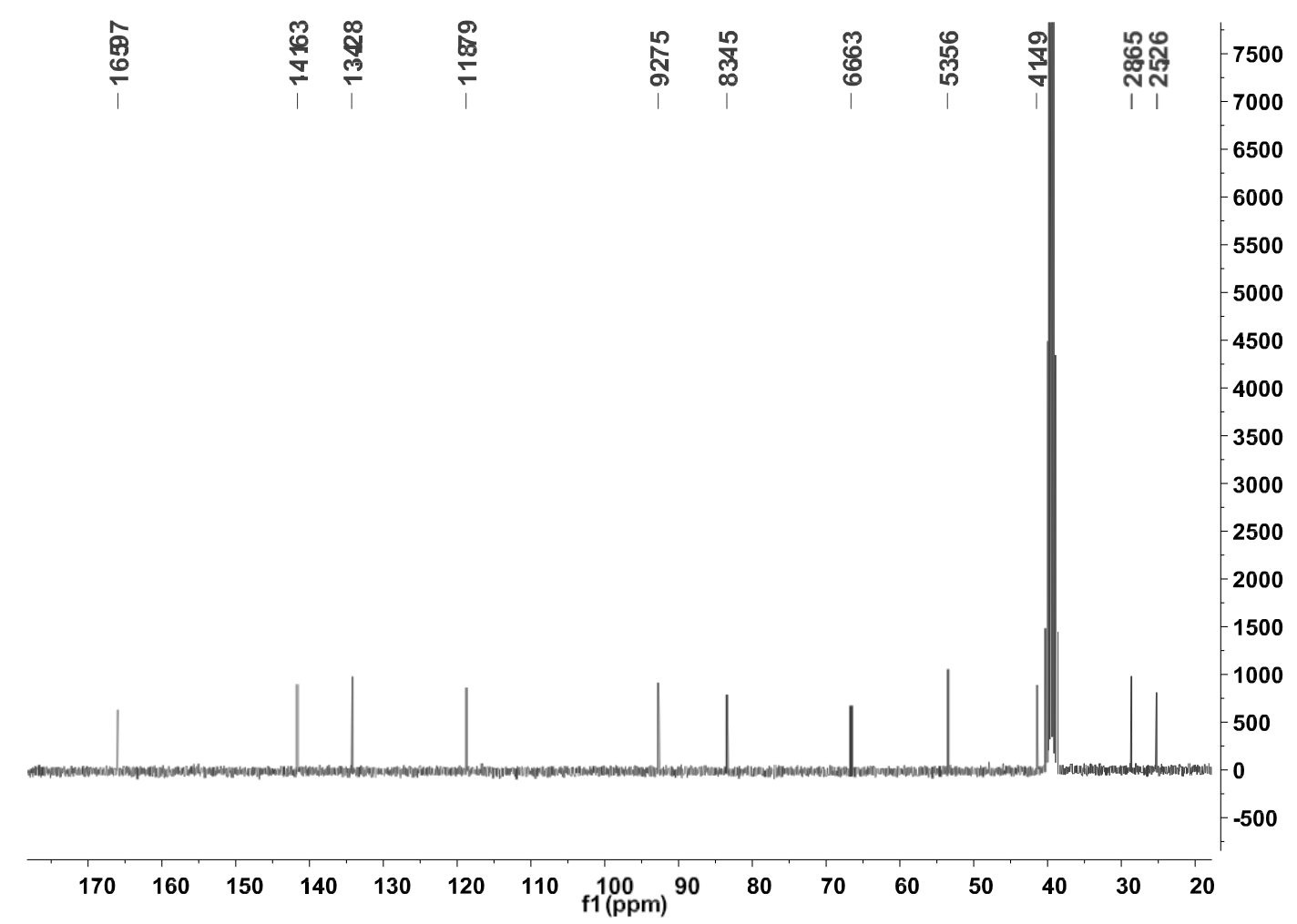

Figure S2. ${ }^{13} \mathrm{C}$ spectrum of compound 1 in DMSO- $d_{6}(75 \mathrm{MHz})$. 


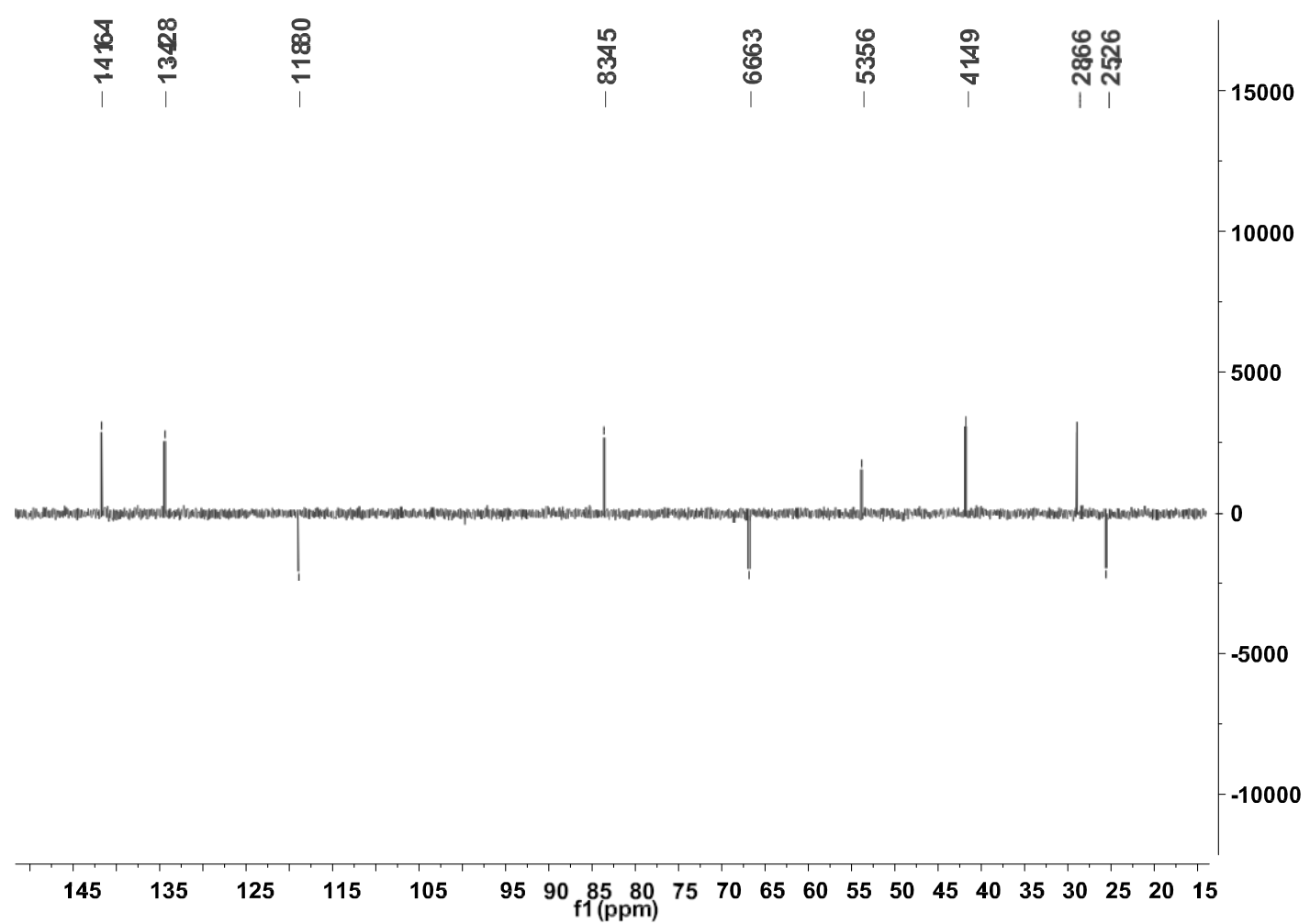

Figure S3. DEPT-135 spectrum of compound 1 in DMSO- $d_{6}(75 \mathrm{MHz})$.

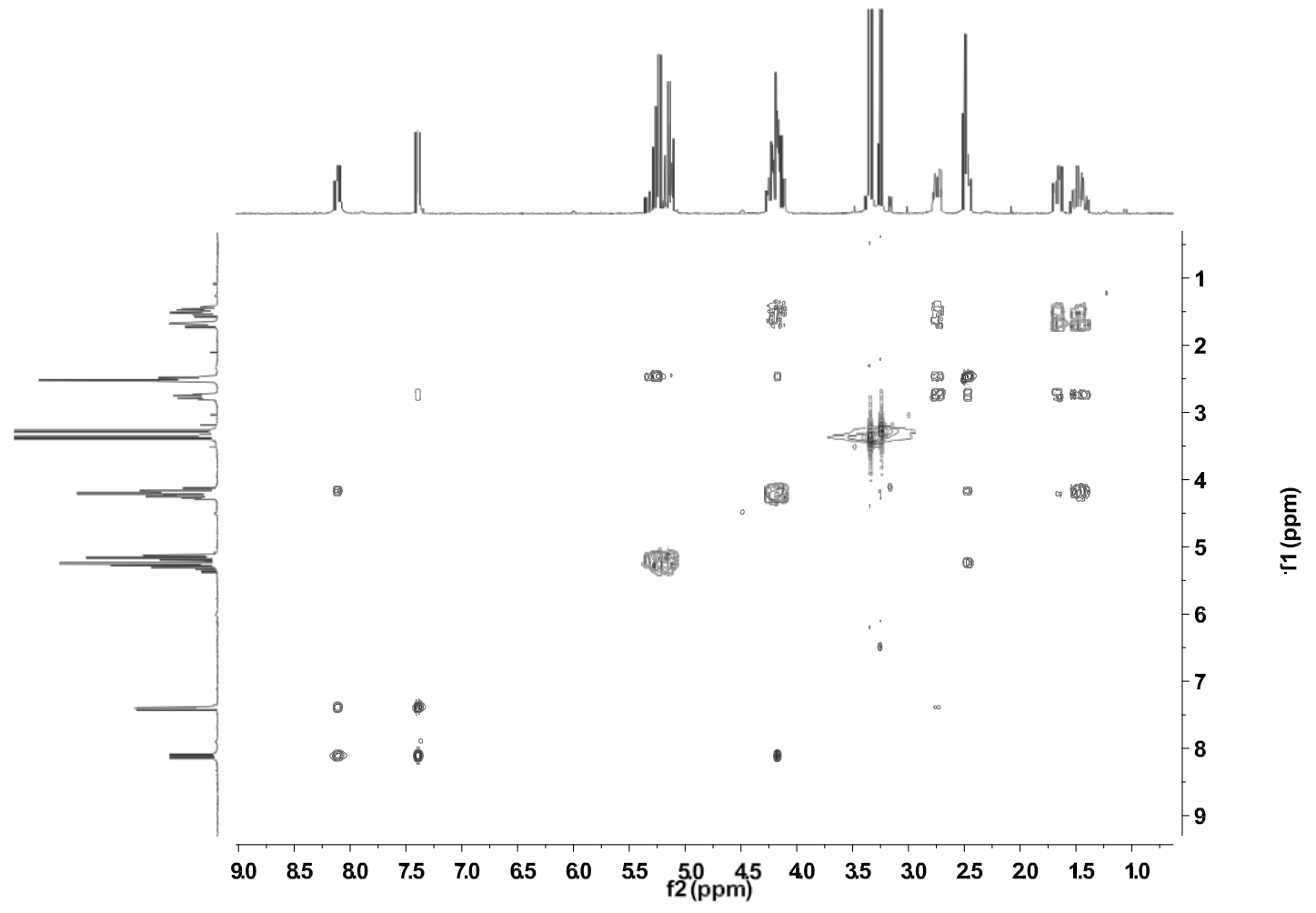

Figure S4. ${ }^{1} \mathrm{H}-{ }^{1} \mathrm{H}$ COSY spectrum of compound $\mathbf{1}$ in DMSO- $d_{6}$. 


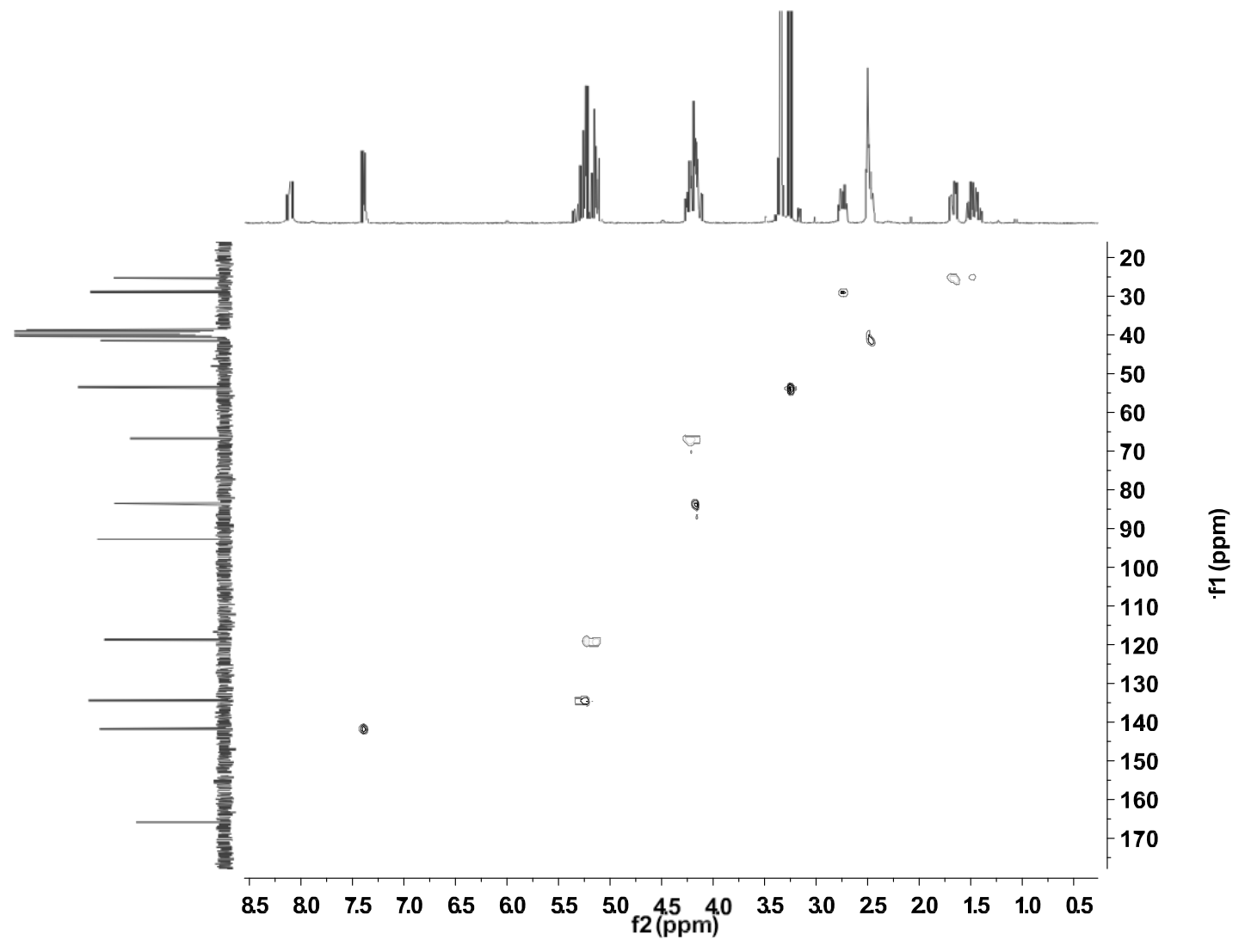

Figure S5. HSQC spectrum of compound $\mathbf{1}$ in DMSO- $d_{6}$.

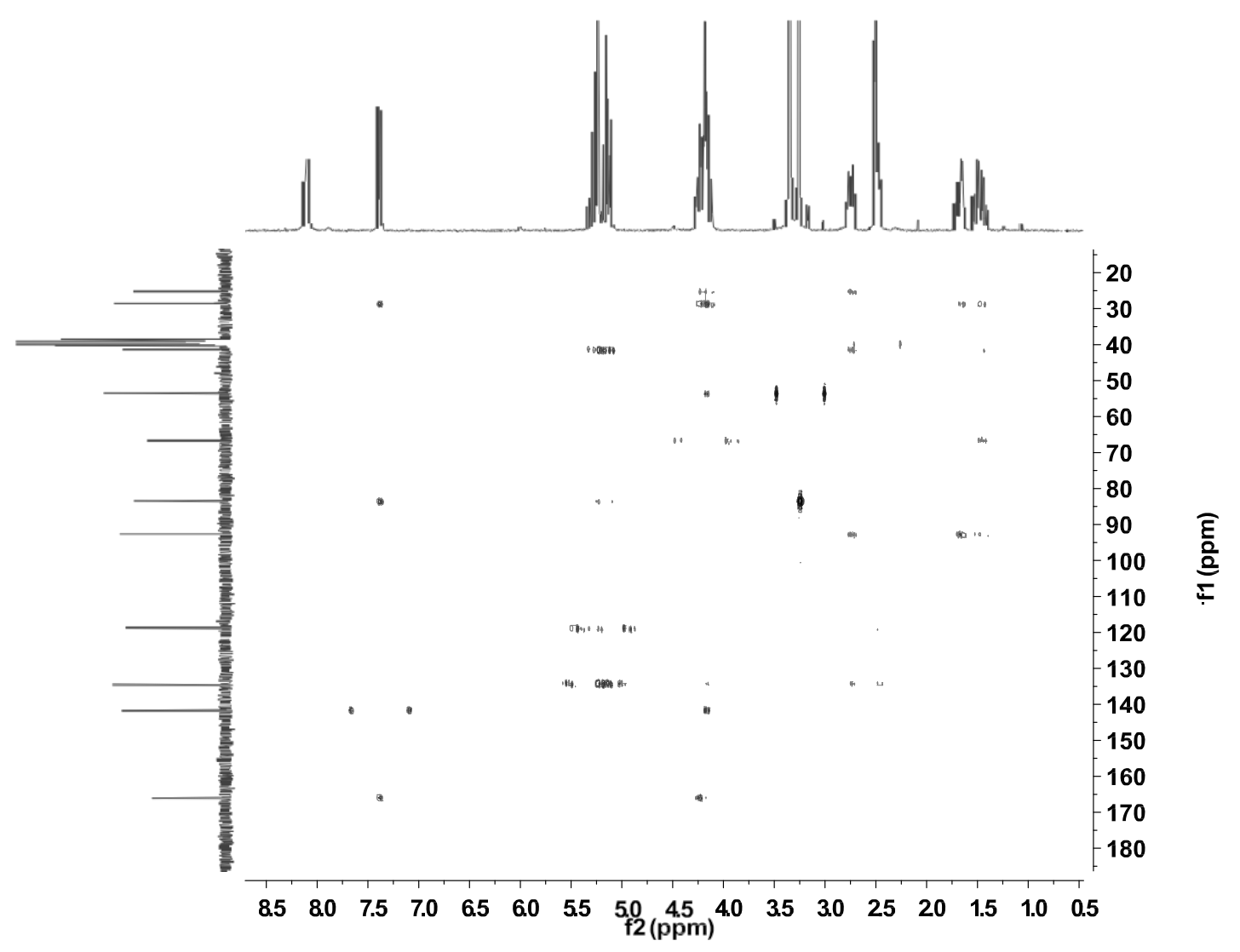

Figure S6. HMBC spectrum of compound 1 in DMSO- $d_{6}$. 


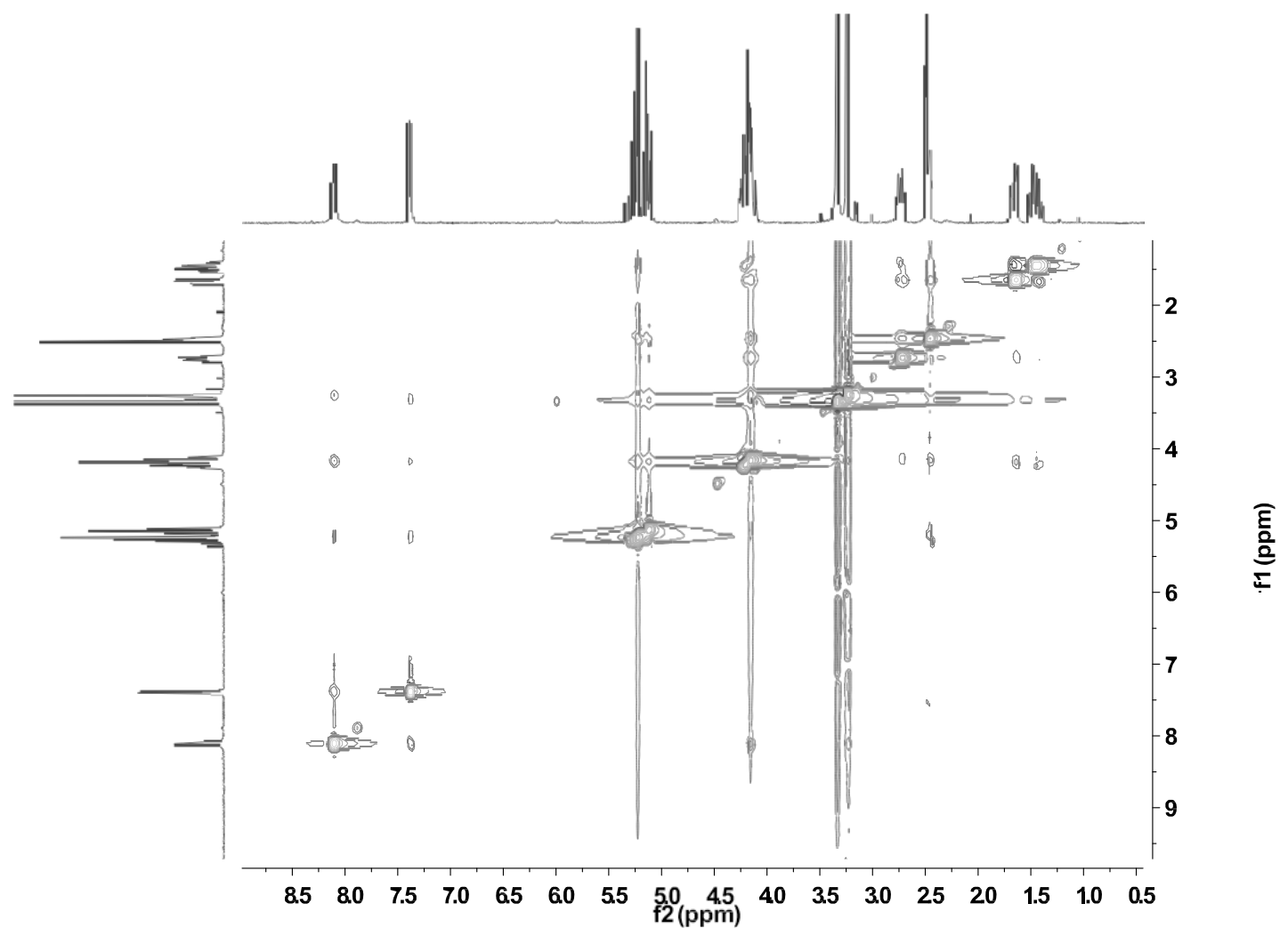

Figure S7. NOESY spectrum of compound $\mathbf{1}$ in DMSO- $d_{6}$.
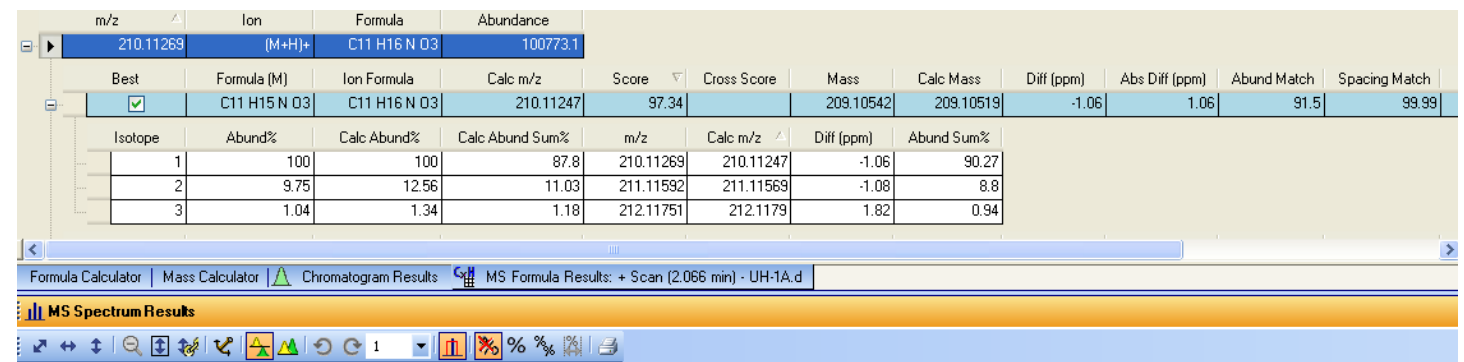

『↔

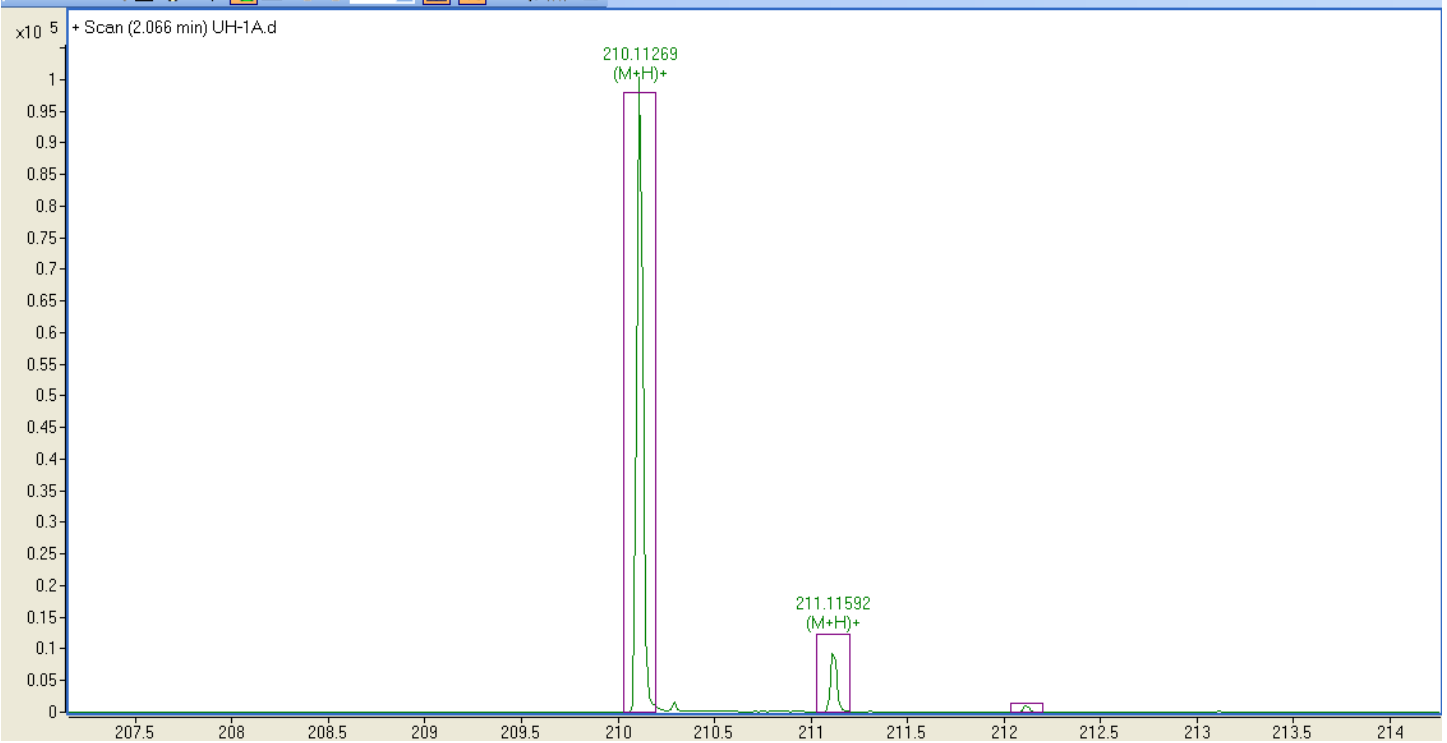

Figure S8. HR-ESI-MS spectrum of $\mathbf{1}$. 


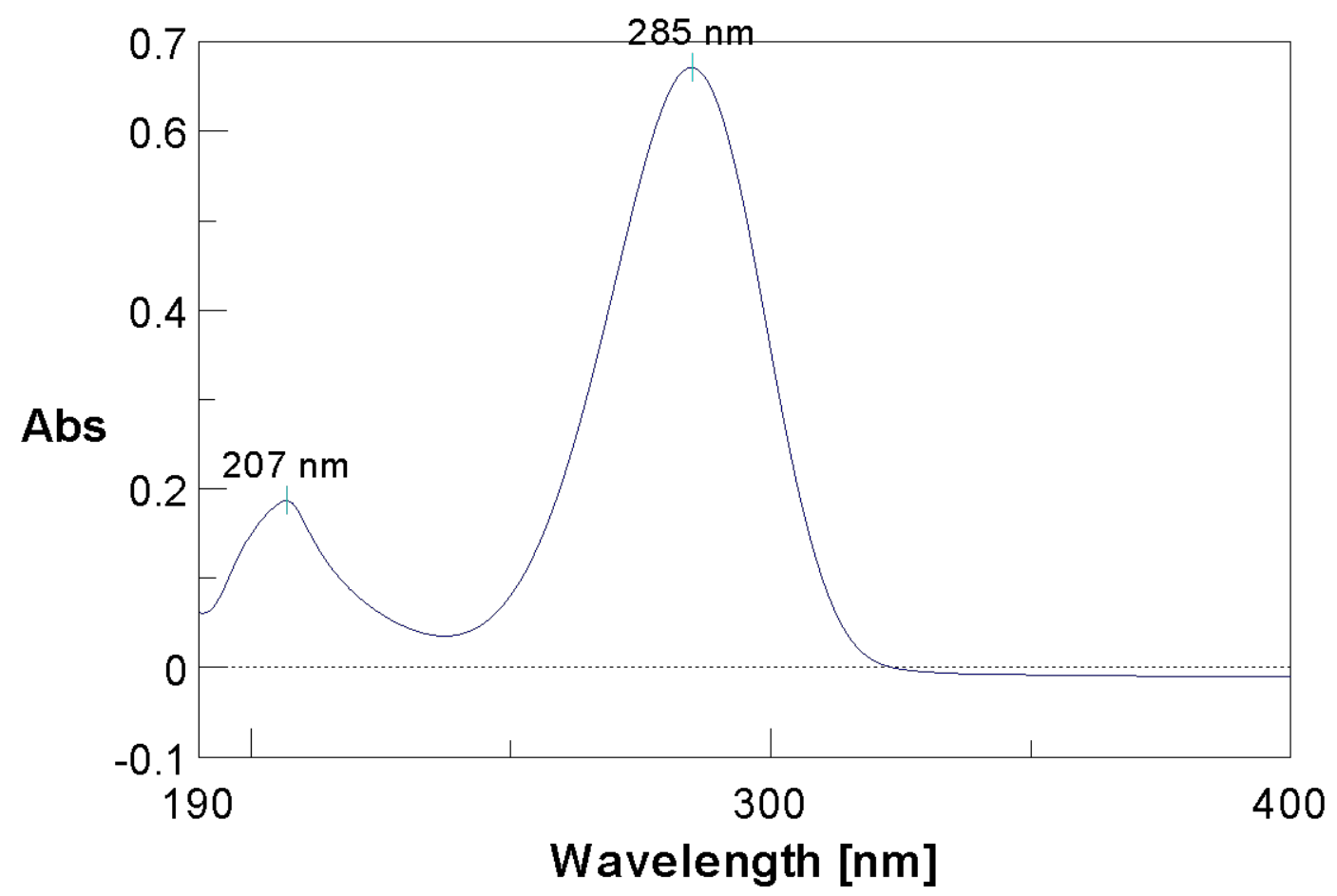

Figure S9. UV spectrum of $\mathbf{1}$ in $\mathrm{CH}_{3} \mathrm{OH}$.

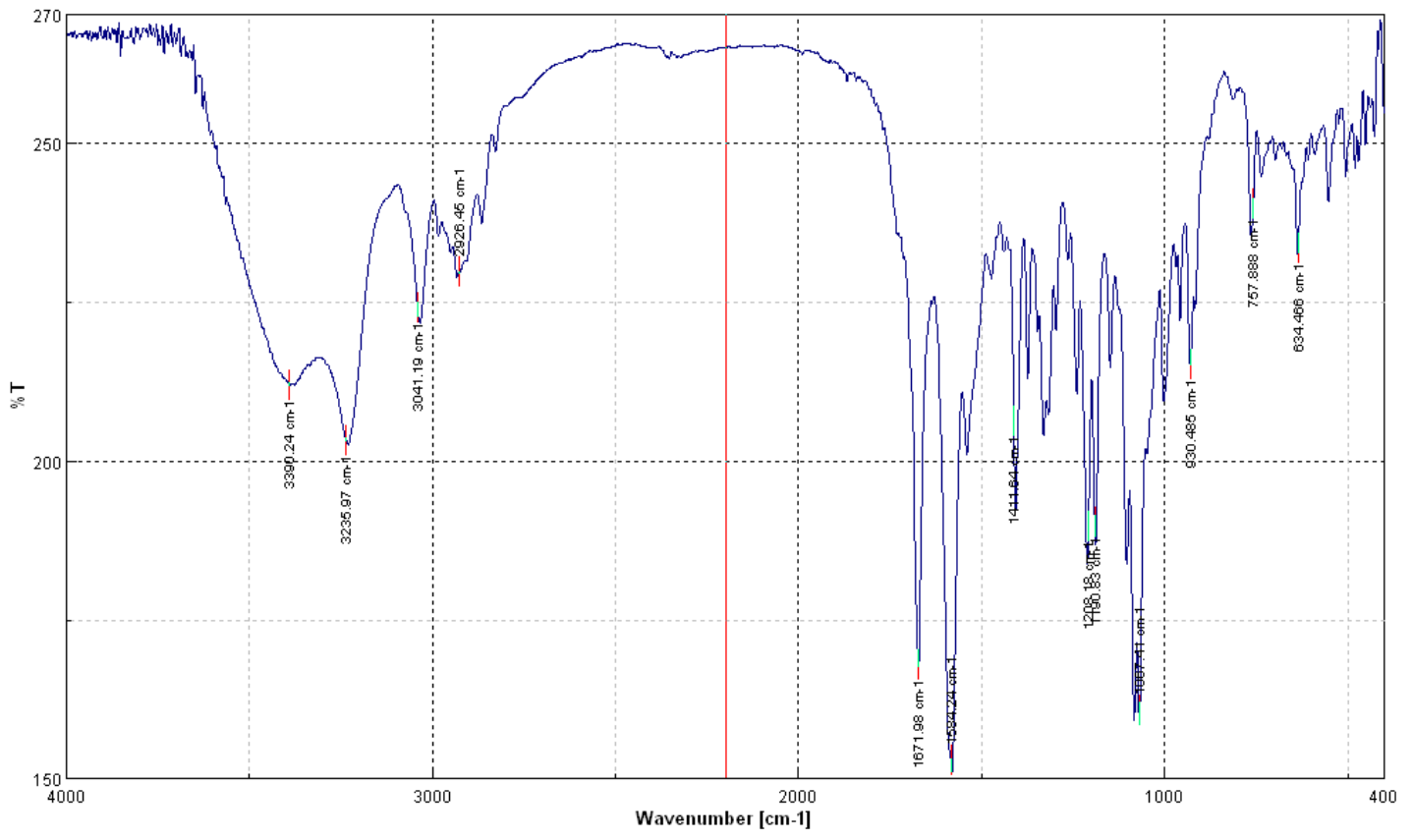

Figure S10. IR ( $\mathrm{KBr}$ disc) spectrum of $\mathbf{1}$. 
Figure S11. Single crystal X-ray data and structures of $\mathbf{1}$.

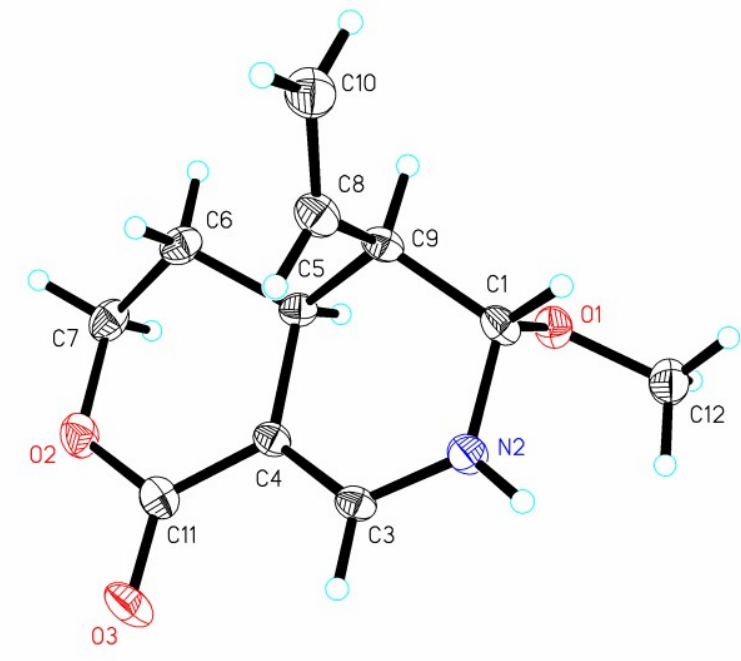

Crystal data of compound $\mathbf{1}$ was deposited in the Cambridge Crystallographic

Data Centre (CCDC 980271).

Table S1. Crystal data and structure refinement for $\mathbf{1}$.

Identification code

Empirical formula

Formula weight

Temperature

Wavelength

Crystal system, space group

Unit cell dimensions

Volume

Z, Calculated density

Absorption coefficient

$\mathrm{F}(000)$

Crystal size

Theta range for data collection

Limiting indices

Reflections collected / unique

Completeness to theta $=62.83$

Absorption correction
1

C11 H15 N O3

209.24

173.02(18) K

$1.5418 \mathrm{~A}$

Orthorhombic, P 212121

$\mathrm{a}=8.2646(2) \mathrm{A} \quad$ alpha $=90$ deg.

$\mathrm{b}=11.2177(3) \mathrm{A} \quad$ beta $=90 \mathrm{deg}$.

$\mathrm{c}=11.7920(3) \mathrm{A} \quad$ gamma $=90 \mathrm{deg}$.

1093.23(5) $\mathrm{A}^{\wedge} 3$

4, $1.198 \mathrm{Mg} / \mathrm{m} \wedge 3$

$0.730 \mathrm{~mm}^{\wedge}-1$

424

$0.32 \times 0.28 \times 0.24 \mathrm{~mm}$

5.44 to $62.83 \mathrm{deg}$.

$-9<=\mathrm{h}<=9,-12<=\mathrm{k}<=12,-13<=\mathrm{l}<=13$

$8820 / 1742$ [R(int) $=0.0294]$

$99.1 \%$

Semi-empirical from equivalents 
Max. and min. transmission

Refinement method

Data / restraints / parameters

Goodness-of-fit on $\mathrm{F}^{\wedge} 2$

Final $\mathrm{R}$ indices $[\mathrm{I}>2 \operatorname{sigma}(\mathrm{I})]$

$\mathrm{R}$ indices (all data)

Absolute structure parameter

Largest diff. peak and hole
1.00000 and 0.70217

Full-matrix least-squares on $\mathrm{F}^{\wedge} 2$

1742 / 0 / 137

1.083

$\mathrm{R} 1=0.0264, \mathrm{wR} 2=0.0693$

$\mathrm{R} 1=0.0271, \mathrm{wR} 2=0.0698$

$-0.2(2)$

0.103 and -0.153 e. $A^{\wedge}-3$

Table S 2. Atomic coordinates ( $\mathrm{x} 10^{\wedge} 4$ ) and equivalent isotropic displacement parameters $\left(\mathrm{A}^{\wedge} 2 \times 10^{\wedge} 3\right)$ for $\mathbf{1} . \mathrm{U}(\mathrm{eq})$ is defined as one third of the trace of the orthogonalized Uij tensor.

\begin{tabular}{lrrrr}
\hline $\mathrm{U}(\mathrm{eq})$ & & $\mathrm{x}$ & $\mathrm{y}$ & $\mathrm{z}$ \\
\hline $\mathrm{N}(2)$ & $8090(2)$ & $8450(1)$ & $3338(1)$ & $32(1)$ \\
$\mathrm{O}(1)$ & $8101(1)$ & $6363(1)$ & $3015(1)$ & $34(1)$ \\
$\mathrm{O}(2)$ & $9294(1)$ & $9434(1)$ & $-522(1)$ & $39(1)$ \\
$\mathrm{O}(3)$ & $7702(2)$ & $10573(1)$ & $490(1)$ & $44(1)$ \\
$\mathrm{C}(1)$ & $9044(2)$ & $7366(1)$ & $3342(1)$ & $29(1)$ \\
$\mathrm{C}(3)$ & $7940(2)$ & $9103(1)$ & $2392(1)$ & $27(1)$ \\
$\mathrm{C}(4)$ & $8661(2)$ & $8826(1)$ & $1392(1)$ & $26(1)$ \\
$\mathrm{C}(5)$ & $9719(2)$ & $7733(1)$ & $1318(1)$ & $26(1)$ \\
$\mathrm{C}(6)$ & $10990(2)$ & $7923(1)$ & $402(1)$ & $34(1)$ \\
$\mathrm{C}(7)$ & $10186(2)$ & $8330(2)$ & $-676(1)$ & $38(1)$ \\
$\mathrm{C}(8)$ & $11637(2)$ & $8394(1)$ & $2866(1)$ & $34(1)$ \\
$\mathrm{C}(9)$ & $10435(2)$ & $7467(1)$ & $2497(1)$ & $29(1)$ \\
$\mathrm{C}(10)$ & $13113(2)$ & $8162(2)$ & $3207(1)$ & $42(1)$ \\
$\mathrm{C}(11)$ & $8502(2)$ & $9652(1)$ & $467(1)$ & $32(1)$ \\
$\mathrm{C}(12)$ & $6890(2)$ & $6053(2)$ & $3819(1)$ & $44(1)$ \\
\hline
\end{tabular}

Table S3. Bond lengths [A] and angles [deg] for $\mathbf{1}$.

$$
\begin{aligned}
& \mathrm{N}(2)-\mathrm{C}(3) \\
& \mathrm{N}(2)-\mathrm{C}(1) \\
& \mathrm{N}(2)-\mathrm{H}(2)
\end{aligned}
$$

1.3400(17)

1.4494(17)

0.8600 


\begin{tabular}{|c|c|}
\hline $\mathrm{O}(1)-\mathrm{C}(1)$ & $1.4214(17)$ \\
\hline $\mathrm{O}(1)-\mathrm{C}(12)$ & $1.4219(18)$ \\
\hline $\mathrm{O}(2)-\mathrm{C}(11)$ & $1.3598(17)$ \\
\hline $\mathrm{O}(2)-\mathrm{C}(7)$ & $1.4527(19)$ \\
\hline $\mathrm{O}(3)-\mathrm{C}(11)$ & $1.2264(18)$ \\
\hline $\mathrm{C}(1)-\mathrm{C}(9)$ & $1.525(2)$ \\
\hline $\mathrm{C}(1)-\mathrm{H}(1)$ & 0.9800 \\
\hline$C(3)-C(4)$ & $1.3566(19)$ \\
\hline C(3)-H(3) & 0.9300 \\
\hline$C(4)-C(11)$ & $1.4374(19)$ \\
\hline$C(4)-C(5)$ & $1.5092(18)$ \\
\hline$C(5)-C(6)$ & $1.5217(19)$ \\
\hline$C(5)-C(9)$ & $1.5403(19)$ \\
\hline $\mathrm{C}(5)-\mathrm{H}(5)$ & 0.9800 \\
\hline C(6)-C(7) & $1.505(2)$ \\
\hline $\mathrm{C}(6)-\mathrm{H}(6 \mathrm{~A})$ & 0.9700 \\
\hline $\mathrm{C}(6)-\mathrm{H}(6 \mathrm{~B})$ & 0.9700 \\
\hline $\mathrm{C}(7)-\mathrm{H}(7 \mathrm{~A})$ & 0.9700 \\
\hline $\mathrm{C}(7)-\mathrm{H}(7 \mathrm{~B})$ & 0.9700 \\
\hline C(8)-C(10) & $1.310(2)$ \\
\hline $\mathrm{C}(8)-\mathrm{C}(9)$ & $1.503(2)$ \\
\hline $\mathrm{C}(8)-\mathrm{H}(8)$ & 0.9300 \\
\hline $\mathrm{C}(9)-\mathrm{H}(9)$ & 0.9800 \\
\hline $\mathrm{C}(10)-\mathrm{H}(10 \mathrm{~A})$ & 0.9300 \\
\hline $\mathrm{C}(10)-\mathrm{H}(10 \mathrm{~B})$ & 0.9300 \\
\hline $\mathrm{C}(12)-\mathrm{H}(12 \mathrm{~A})$ & 0.9600 \\
\hline $\mathrm{C}(12)-\mathrm{H}(12 \mathrm{~B})$ & 0.9600 \\
\hline $\mathrm{C}(12)-\mathrm{H}(12 \mathrm{C})$ & 0.9600 \\
\hline $\mathrm{C}(3)-\mathrm{N}(2)-\mathrm{C}(1)$ & $120.77(11)$ \\
\hline $\mathrm{C}(3)-\mathrm{N}(2)-\mathrm{H}(2)$ & 119.6 \\
\hline $\mathrm{C}(1)-\mathrm{N}(2)-\mathrm{H}(2)$ & 119.6 \\
\hline $\mathrm{C}(1)-\mathrm{O}(1)-\mathrm{C}(12)$ & $113.52(11)$ \\
\hline $\mathrm{C}(11)-\mathrm{O}(2)-\mathrm{C}(7)$ & 120.31(11) \\
\hline $\mathrm{O}(1)-\mathrm{C}(1)-\mathrm{N}(2)$ & 111.37(11) \\
\hline $\mathrm{O}(1)-\mathrm{C}(1)-\mathrm{C}(9)$ & $107.16(10)$ \\
\hline $\mathrm{N}(2)-\mathrm{C}(1)-\mathrm{C}(9)$ & $110.21(11)$ \\
\hline $\mathrm{O}(1)-\mathrm{C}(1)-\mathrm{H}(1)$ & 109.4 \\
\hline $\mathrm{N}(2)-\mathrm{C}(1)-\mathrm{H}(1)$ & 109.4 \\
\hline $\mathrm{C}(9)-\mathrm{C}(1)-\mathrm{H}(1)$ & 109.4 \\
\hline $\mathrm{N}(2)-\mathrm{C}(3)-\mathrm{C}(4)$ & $123.89(12)$ \\
\hline $\mathrm{N}(2)-\mathrm{C}(3)-\mathrm{H}(3)$ & 118.1 \\
\hline C(4)-C(3)-H(3) & 118.1 \\
\hline $\mathrm{C}(3)-\mathrm{C}(4)-\mathrm{C}(11)$ & $118.14(12)$ \\
\hline
\end{tabular}




\begin{tabular}{|c|c|}
\hline C(3)-C(4)-C(5) & $119.41(12)$ \\
\hline$C(11)-C(4)-C(5)$ & $122.20(12)$ \\
\hline$C(4)-C(5)-C(6)$ & 109.10(11) \\
\hline$C(4)-C(5)-C(9)$ & $109.08(10)$ \\
\hline$C(6)-C(5)-C(9)$ & $113.77(11)$ \\
\hline $\mathrm{C}(4)-\mathrm{C}(5)-\mathrm{H}(5)$ & 108.3 \\
\hline $\mathrm{C}(6)-\mathrm{C}(5)-\mathrm{H}(5)$ & 108.3 \\
\hline $\mathrm{C}(9)-\mathrm{C}(5)-\mathrm{H}(5)$ & 108.3 \\
\hline $\mathrm{C}(7)-\mathrm{C}(6)-\mathrm{C}(5)$ & $109.70(12)$ \\
\hline $\mathrm{C}(7)-\mathrm{C}(6)-\mathrm{H}(6 \mathrm{~A})$ & 109.7 \\
\hline $\mathrm{C}(5)-\mathrm{C}(6)-\mathrm{H}(6 \mathrm{~A})$ & 109.7 \\
\hline $\mathrm{C}(7)-\mathrm{C}(6)-\mathrm{H}(6 \mathrm{~B})$ & 109.7 \\
\hline C(5)-C(6)-H(6B) & 109.7 \\
\hline $\mathrm{H}(6 \mathrm{~A})-\mathrm{C}(6)-\mathrm{H}(6 \mathrm{~B})$ & 108.2 \\
\hline $\mathrm{O}(2)-\mathrm{C}(7)-\mathrm{C}(6)$ & $112.18(12)$ \\
\hline $\mathrm{O}(2)-\mathrm{C}(7)-\mathrm{H}(7 \mathrm{~A})$ & 109.2 \\
\hline $\mathrm{C}(6)-\mathrm{C}(7)-\mathrm{H}(7 \mathrm{~A})$ & 109.2 \\
\hline $\mathrm{O}(2)-\mathrm{C}(7)-\mathrm{H}(7 \mathrm{~B})$ & 109.2 \\
\hline C(6)-C(7)-H(7B) & 109.2 \\
\hline $\mathrm{H}(7 \mathrm{~A})-\mathrm{C}(7)-\mathrm{H}(7 \mathrm{~B})$ & 107.9 \\
\hline C(10)-C(8)-C(9) & $124.57(14)$ \\
\hline $\mathrm{C}(10)-\mathrm{C}(8)-\mathrm{H}(8)$ & 117.7 \\
\hline C(9)-C(8)-H(8) & 117.7 \\
\hline $\mathrm{C}(8)-\mathrm{C}(9)-\mathrm{C}(1)$ & $111.14(11)$ \\
\hline $\mathrm{C}(8)-\mathrm{C}(9)-\mathrm{C}(5)$ & $112.45(11)$ \\
\hline $\mathrm{C}(1)-\mathrm{C}(9)-\mathrm{C}(5)$ & $108.35(11)$ \\
\hline $\mathrm{C}(8)-\mathrm{C}(9)-\mathrm{H}(9)$ & 108.3 \\
\hline $\mathrm{C}(1)-\mathrm{C}(9)-\mathrm{H}(9)$ & 108.3 \\
\hline $\mathrm{C}(5)-\mathrm{C}(9)-\mathrm{H}(9)$ & 108.3 \\
\hline $\mathrm{C}(8)-\mathrm{C}(10)-\mathrm{H}(10 \mathrm{~A})$ & 120.0 \\
\hline C(8)-C(10)-H(10B) & 120.0 \\
\hline $\mathrm{H}(10 \mathrm{~A})-\mathrm{C}(10)-\mathrm{H}(10 \mathrm{~B})$ & 120.0 \\
\hline $\mathrm{O}(3)-\mathrm{C}(11)-\mathrm{O}(2)$ & $115.42(12)$ \\
\hline $\mathrm{O}(3)-\mathrm{C}(11)-\mathrm{C}(4)$ & $125.17(13)$ \\
\hline $\mathrm{O}(2)-\mathrm{C}(11)-\mathrm{C}(4)$ & $119.41(12)$ \\
\hline $\mathrm{O}(1)-\mathrm{C}(12)-\mathrm{H}(12 \mathrm{~A})$ & 109.5 \\
\hline $\mathrm{O}(1)-\mathrm{C}(12)-\mathrm{H}(12 \mathrm{~B})$ & 109.5 \\
\hline $\mathrm{H}(12 \mathrm{~A})-\mathrm{C}(12)-\mathrm{H}(12 \mathrm{~B})$ & 109.5 \\
\hline $\mathrm{O}(1)-\mathrm{C}(12)-\mathrm{H}(12 \mathrm{C})$ & 109.5 \\
\hline $\mathrm{H}(12 \mathrm{~A})-\mathrm{C}(12)-\mathrm{H}(12 \mathrm{C})$ & 109.5 \\
\hline $\mathrm{H}(12 \mathrm{~B})-\mathrm{C}(12)-\mathrm{H}(12 \mathrm{C})$ & 109.5 \\
\hline
\end{tabular}

Symmetry transformations used to generate equivalent atoms: 
Table S 4. Anisotropic displacement parameters $\left(\mathrm{A}^{\wedge} 2 \times 10^{\wedge} 3\right)$ for $\mathbf{1}$. The anisotropic displacement factor exponent takes the form: $-2 \mathrm{pi} \wedge 2\left[\mathrm{~h} \wedge 2 \mathrm{a}^{* \wedge} 2 \mathrm{U} 11+\ldots+2 \mathrm{~h} \mathrm{k} \mathrm{a*}\right.$ b* U12 ]

\begin{tabular}{lrrrrrr}
\hline U11 & U22 & U33 & U23 & U13 & U12 \\
& & & & & & \\
\hline $\mathrm{N}(2)$ & $44(1)$ & $29(1)$ & $24(1)$ & $-2(1)$ & $4(1)$ & $9(1)$ \\
O(1) & $42(1)$ & $28(1)$ & $31(1)$ & $-1(1)$ & $3(1)$ & $0(1)$ \\
O(2) & $48(1)$ & $43(1)$ & $26(1)$ & $6(1)$ & $1(1)$ & $1(1)$ \\
O(3) & $66(1)$ & $33(1)$ & $34(1)$ & $4(1)$ & $-8(1)$ & $14(1)$ \\
C(1) & $39(1)$ & $25(1)$ & $24(1)$ & $0(1)$ & $-5(1)$ & $4(1)$ \\
C(3) & $31(1)$ & $23(1)$ & $28(1)$ & $-4(1)$ & $-3(1)$ & $5(1)$ \\
C(4) & $28(1)$ & $24(1)$ & $25(1)$ & $-1(1)$ & $-4(1)$ & $2(1)$ \\
C(5) & $27(1)$ & $25(1)$ & $26(1)$ & $-5(1)$ & $-1(1)$ & $3(1)$ \\
C(6) & $32(1)$ & $42(1)$ & $30(1)$ & $-6(1)$ & $3(1)$ & $3(1)$ \\
C(7) & $37(1)$ & $50(1)$ & $27(1)$ & $-4(1)$ & $6(1)$ & $-1(1)$ \\
C(8) & $40(1)$ & $32(1)$ & $31(1)$ & $-1(1)$ & $-5(1)$ & $2(1)$ \\
C(9) & $33(1)$ & $24(1)$ & $29(1)$ & $-2(1)$ & $-4(1)$ & $7(1)$ \\
C(10) & $40(1)$ & $49(1)$ & $39(1)$ & $2(1)$ & $-8(1)$ & $-3(1)$ \\
C(11) & $38(1)$ & $32(1)$ & $26(1)$ & $-1(1)$ & $-7(1)$ & $-2(1)$ \\
C(12) & $49(1)$ & $42(1)$ & $42(1)$ & $5(1)$ & $7(1)$ & $0(1)$ \\
& & & & & & \\
\hline
\end{tabular}

Table S5. Hydrogen coordinates ( $\mathrm{x} 10^{\wedge} 4$ ) and isotropic displacement parameters $\left(\mathrm{A}^{\wedge} 2 \times 10^{\wedge} 3\right)$ for 1 .

\begin{tabular}{lcccc}
\hline & $x$ & $y$ & $z$ & $U(e q)$ \\
& & & & \\
$H(2)$ & 7613 & 8676 & 3949 & 38 \\
H(1) & 9483 & 7234 & 4104 & 35 \\
H(3) & 7305 & 9787 & 2423 & 33 \\
H(5) & 9040 & 7056 & 1094 & 31 \\
H(6A) & 11764 & 8518 & 650 & 41 \\
H(6B) & 11569 & 7185 & 266 & 41 \\
H(7A) & 11003 & 8445 & -1256 & 46 \\
H(7B) & 9450 & 7715 & -936 & 46 \\
H(8) & 11311 & 9187 & 2852 & 41 \\
H(9) & 10989 & 6696 & 2463 & 34 \\
H(10A) & 13479 & 7379 & 3232 & 51 \\
H(10B) & 13794 & 8781 & 3424 & 51
\end{tabular}




$\begin{array}{lllll}\mathrm{H}(12 \mathrm{~A}) & 7393 & 5843 & 4525 & 66 \\ \mathrm{H}(12 \mathrm{~B}) & 6279 & 5387 & 3541 & 66 \\ \mathrm{H}(12 \mathrm{C}) & 6180 & 6720 & 3934 & 66\end{array}$

Table S6. Torsion angles [deg] for $\mathbf{1}$.

\begin{tabular}{|c|c|}
\hline $\mathrm{C}(12)-\mathrm{O}(1)-\mathrm{C}(1)-\mathrm{N}(2)$ & $-67.92(14)$ \\
\hline $\mathrm{C}(12)-\mathrm{O}(1)-\mathrm{C}(1)-\mathrm{C}(9)$ & $171.47(11)$ \\
\hline $\mathrm{C}(3)-\mathrm{N}(2)-\mathrm{C}(1)-\mathrm{O}(1)$ & $-87.87(15)$ \\
\hline $\mathrm{C}(3)-\mathrm{N}(2)-\mathrm{C}(1)-\mathrm{C}(9)$ & 30.93(17) \\
\hline C(1)-N(2)-C(3)-C(4) & $-0.8(2)$ \\
\hline $\mathrm{N}(2)-\mathrm{C}(3)-\mathrm{C}(4)-\mathrm{C}(11)$ & $-175.30(13)$ \\
\hline N(2)-C(3)-C(4)-C(5) & $-0.9(2)$ \\
\hline C(3)-C(4)-C(5)-C(6) & $-152.01(13)$ \\
\hline $\mathrm{C}(11)-\mathrm{C}(4)-\mathrm{C}(5)-\mathrm{C}(6)$ & 22.18(17) \\
\hline C(3)-C(4)-C(5)-C(9) & $-27.19(17)$ \\
\hline $\mathrm{C}(11)-\mathrm{C}(4)-\mathrm{C}(5)-\mathrm{C}(9)$ & $147.00(13)$ \\
\hline C(4)-C(5)-C(6)-C(7) & $-50.68(15)$ \\
\hline C(9)-C(5)-C(6)-C(7) & $-172.72(12)$ \\
\hline $\mathrm{C}(11)-\mathrm{O}(2)-\mathrm{C}(7)-\mathrm{C}(6)$ & $-35.89(18)$ \\
\hline $\mathrm{C}(5)-\mathrm{C}(6)-\mathrm{C}(7)-\mathrm{O}(2)$ & $59.20(16)$ \\
\hline $\mathrm{C}(10)-\mathrm{C}(8)-\mathrm{C}(9)-\mathrm{C}(1)$ & $111.52(16)$ \\
\hline $\mathrm{C}(10)-\mathrm{C}(8)-\mathrm{C}(9)-\mathrm{C}(5)$ & $-126.81(16)$ \\
\hline $\mathrm{O}(1)-\mathrm{C}(1)-\mathrm{C}(9)-\mathrm{C}(8)$ & $-171.80(10)$ \\
\hline $\mathrm{N}(2)-\mathrm{C}(1)-\mathrm{C}(9)-\mathrm{C}(8)$ & 66.86(14) \\
\hline $\mathrm{O}(1)-\mathrm{C}(1)-\mathrm{C}(9)-\mathrm{C}(5)$ & $64.17(12)$ \\
\hline $\mathrm{N}(2)-\mathrm{C}(1)-\mathrm{C}(9)-\mathrm{C}(5)$ & $-57.17(14)$ \\
\hline$C(4)-C(5)-C(9)-C(8)$ & $-68.45(14)$ \\
\hline $\mathrm{C}(6)-\mathrm{C}(5)-\mathrm{C}(9)-\mathrm{C}(8)$ & 53.60(15) \\
\hline $\mathrm{C}(4)-\mathrm{C}(5)-\mathrm{C}(9)-\mathrm{C}(1)$ & $54.80(14)$ \\
\hline C(6)-C(5)-C(9)-C(1) & $176.84(11)$ \\
\hline $\mathrm{C}(7)-\mathrm{O}(2)-\mathrm{C}(11)-\mathrm{O}(3)$ & $-175.65(13)$ \\
\hline $\mathrm{C}(7)-\mathrm{O}(2)-\mathrm{C}(11)-\mathrm{C}(4)$ & 4.98(19) \\
\hline $\mathrm{C}(3)-\mathrm{C}(4)-\mathrm{C}(11)-\mathrm{O}(3)$ & $-3.4(2)$ \\
\hline $\mathrm{C}(5)-\mathrm{C}(4)-\mathrm{C}(11)-\mathrm{O}(3)$ & $-177.63(14)$ \\
\hline $\mathrm{C}(3)-\mathrm{C}(4)-\mathrm{C}(11)-\mathrm{O}(2)$ & 175.94(13) \\
\hline $\mathrm{C}(5)-\mathrm{C}(4)-\mathrm{C}(11)-\mathrm{O}(2)$ & $1.7(2)$ \\
\hline
\end{tabular}

Symmetry transformations used to generate equivalent atoms: 
Table S 7. Hydrogen bonds for $\mathbf{1}$ [A and deg.].

D-H...A $\mathrm{d}(\mathrm{D}-\mathrm{H})$

$\mathrm{d}(\mathrm{H} . . . \mathrm{A}) \quad \mathrm{d}(\mathrm{D} . . \mathrm{A})$

$<(\mathrm{DHA})$ 


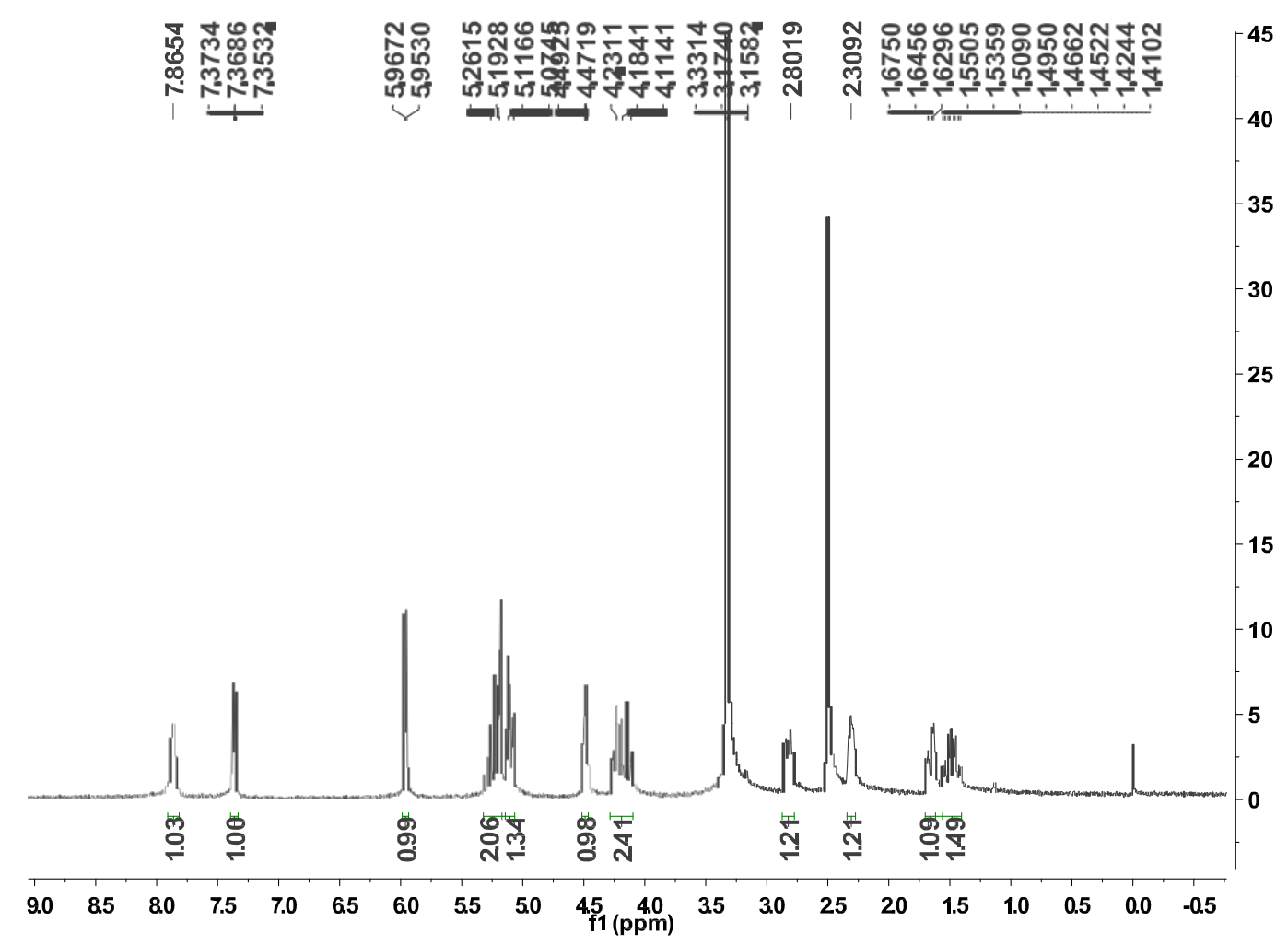

Figure S12. ${ }^{1} \mathrm{H}$ NMR spectrum of compound 2 in DMSO- $d_{6}(300 \mathrm{MHz})$.

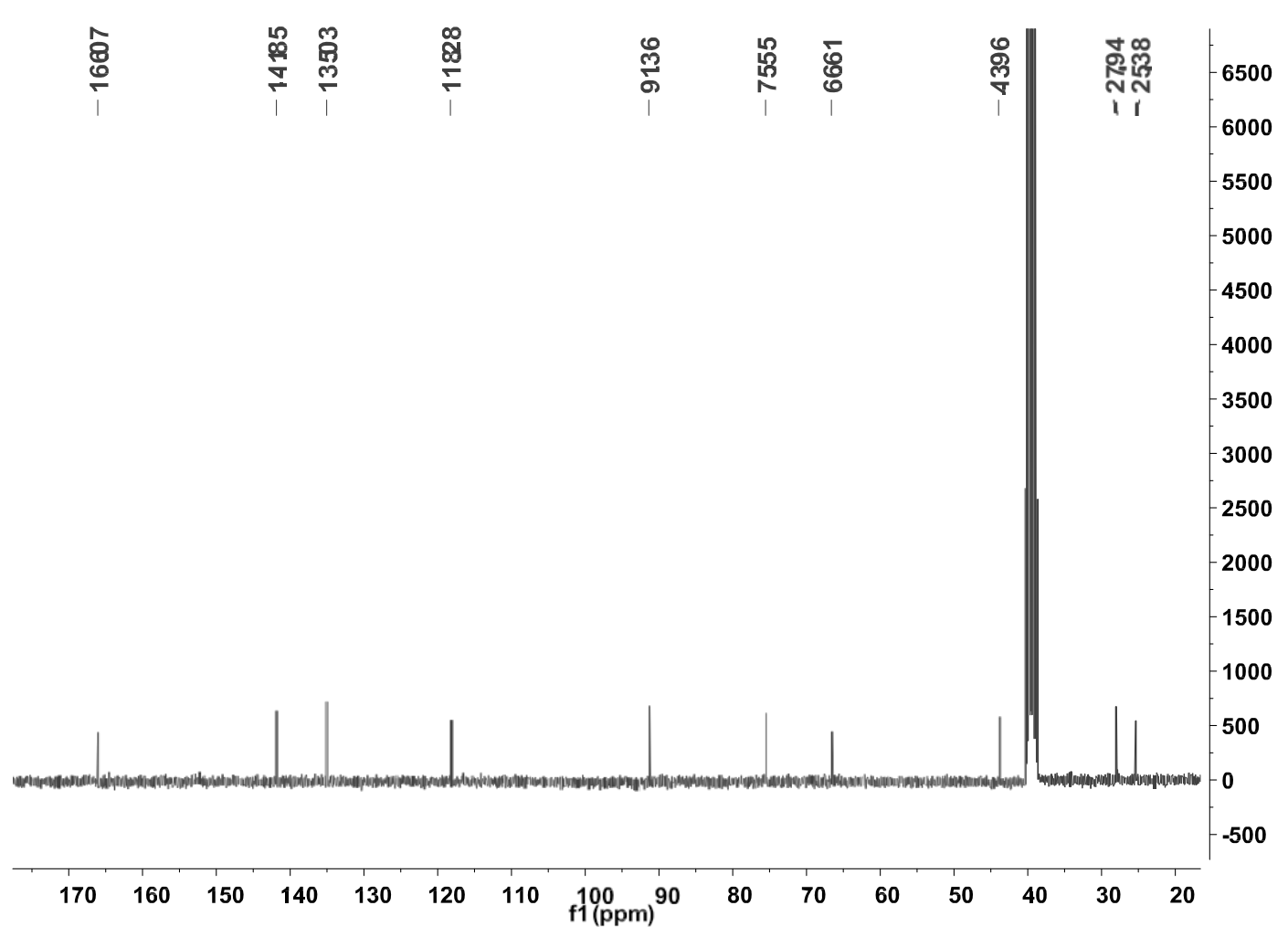

Figure S13. ${ }^{13} \mathrm{C}$ spectrum of compound 2 in DMSO-d $d_{6}(75 \mathrm{MHz})$. 


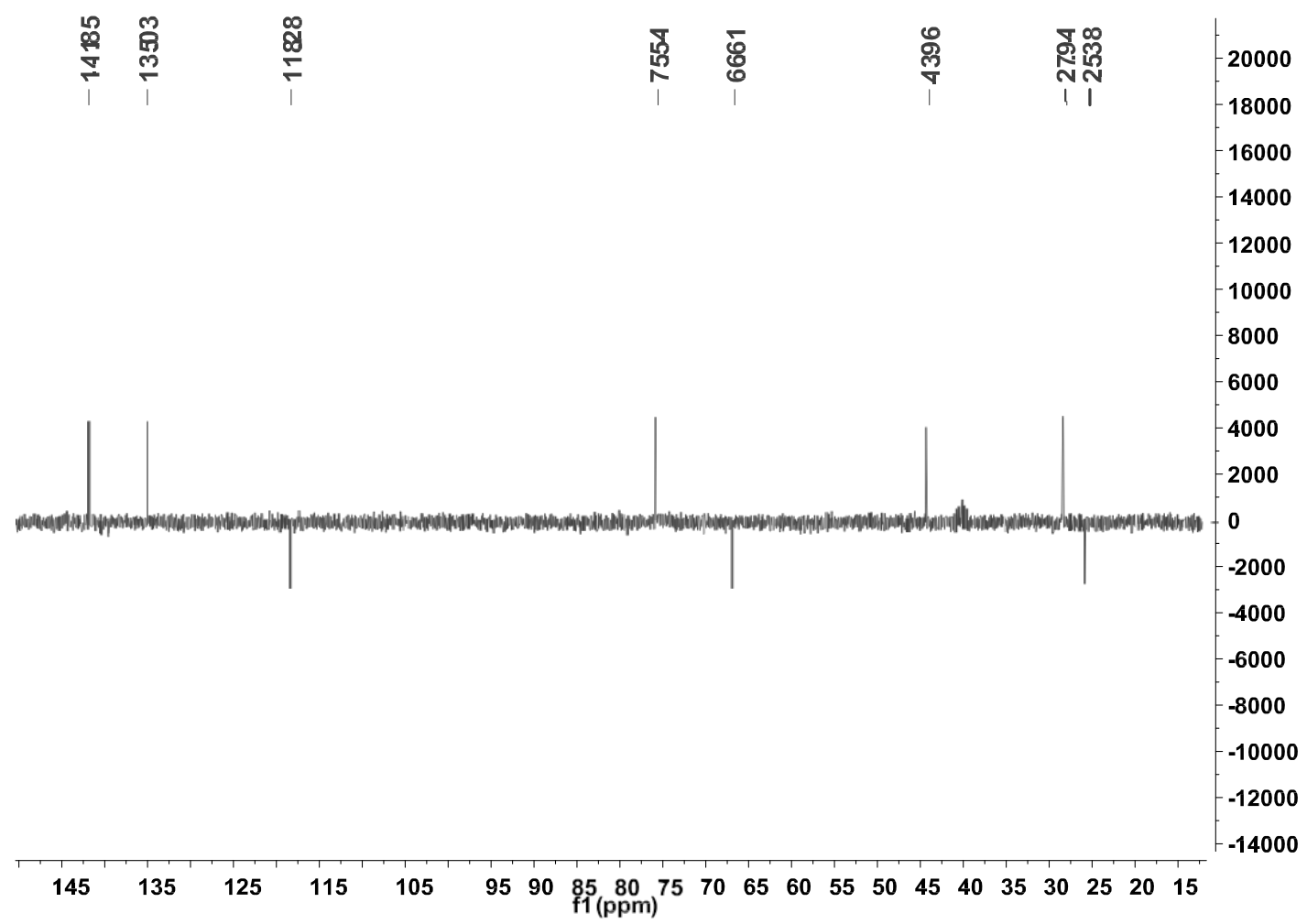

Figure S14. DEPT-135 spectrum of compound 2 in DMSO- $d_{6}(75 \mathrm{MHz})$.

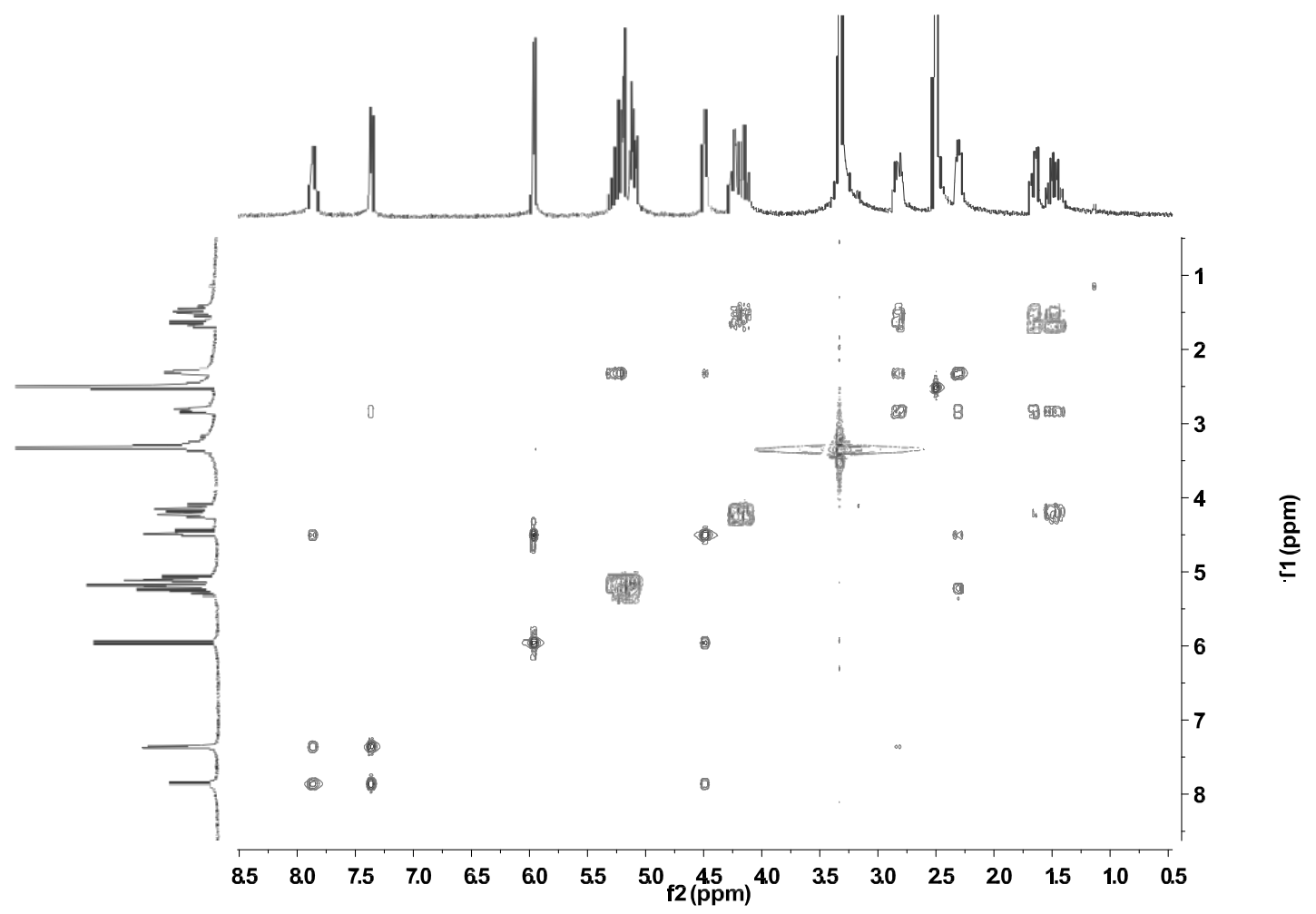

Figure S15. ${ }^{1} \mathrm{H}-{ }^{1} \mathrm{H}$ COSY spectrum of compound 2 in DMSO- $d_{6}$. 


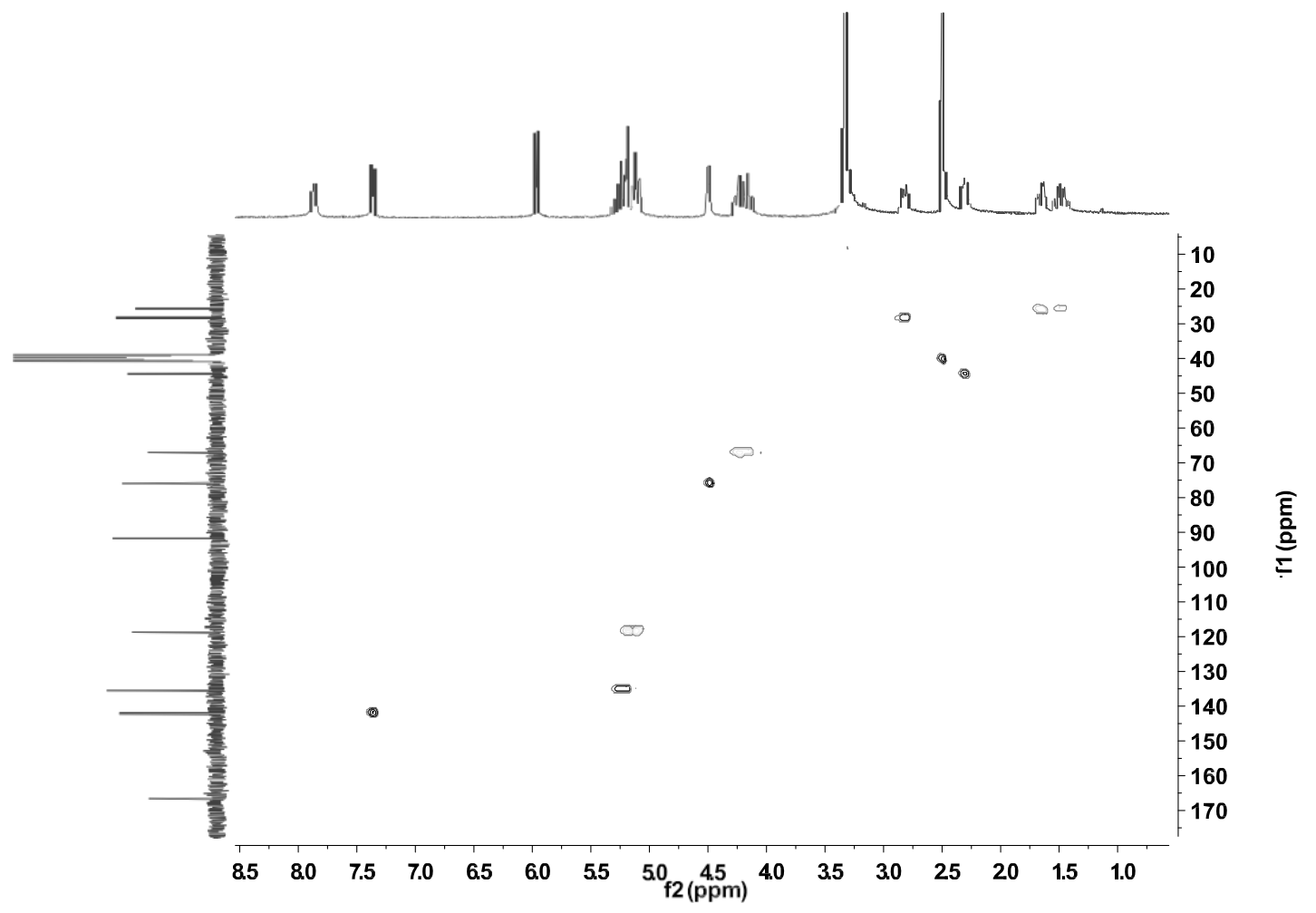

Figure S16. HSQC spectrum of compound 2 in DMSO- $d_{6}$.

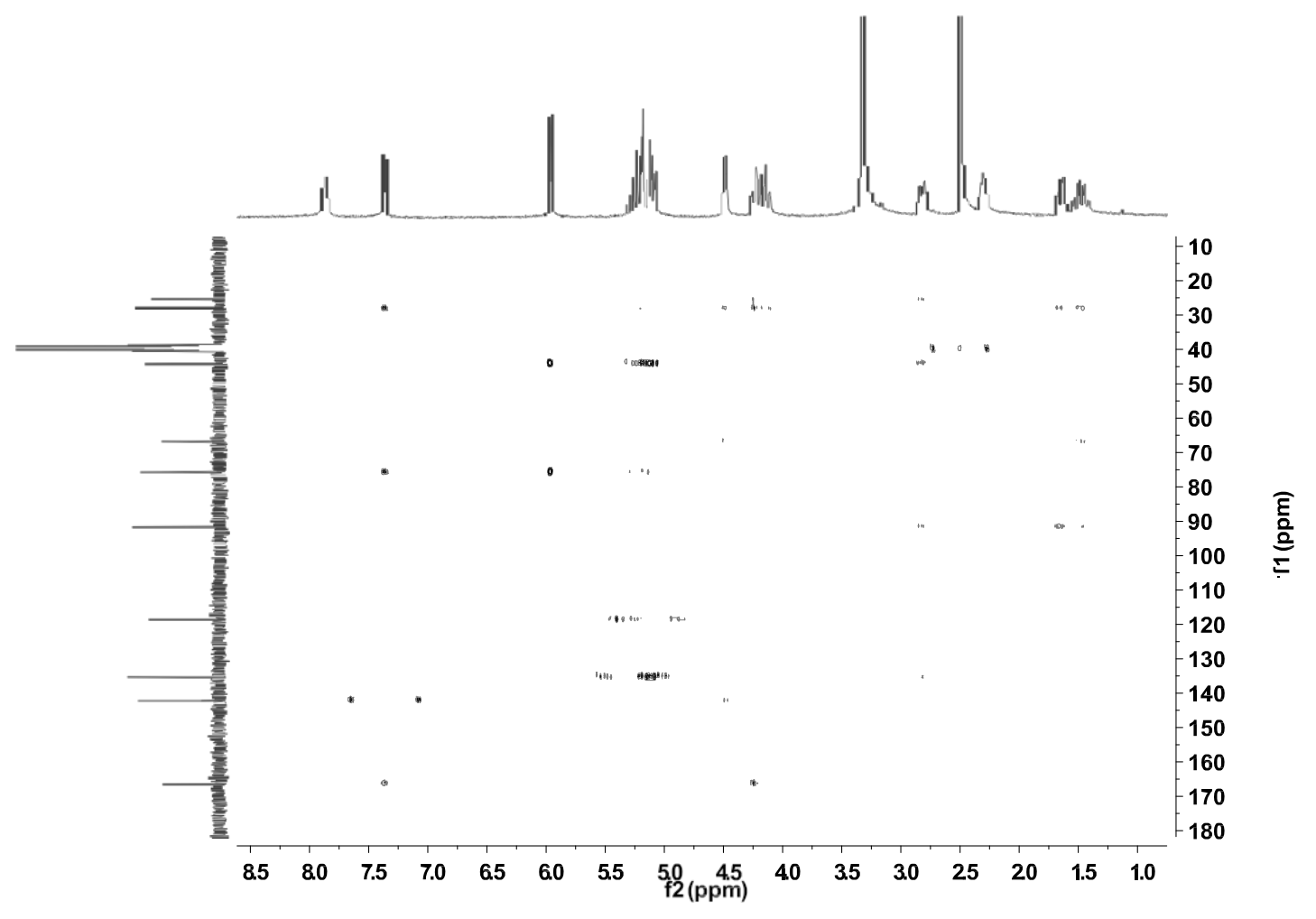

Figure S17. HMBC spectrum of compound 2 in DMSO- $d_{6}$. 


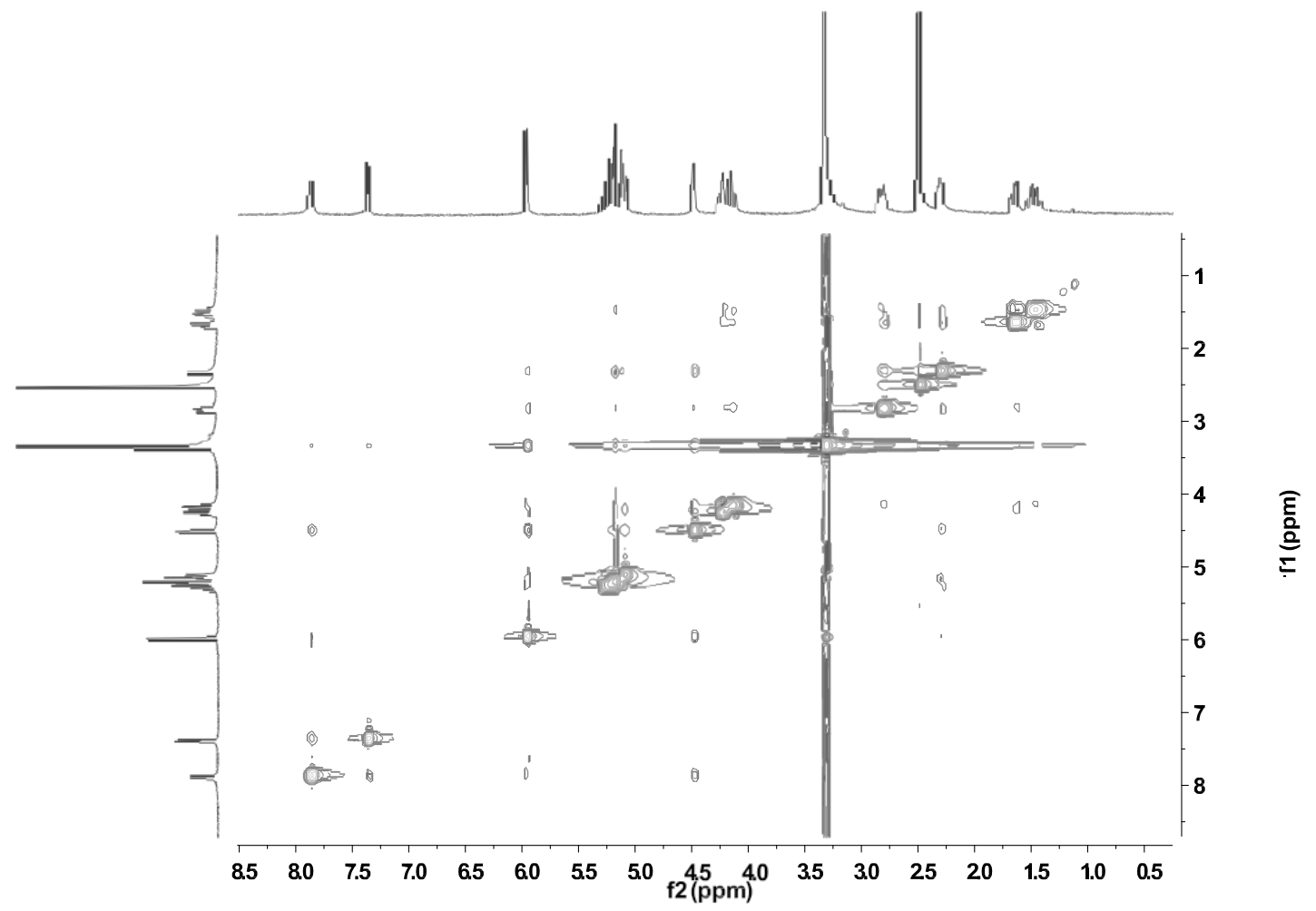

Figure S18. NOESY spectrum of compound 2 in DMSO- $d_{6}$.

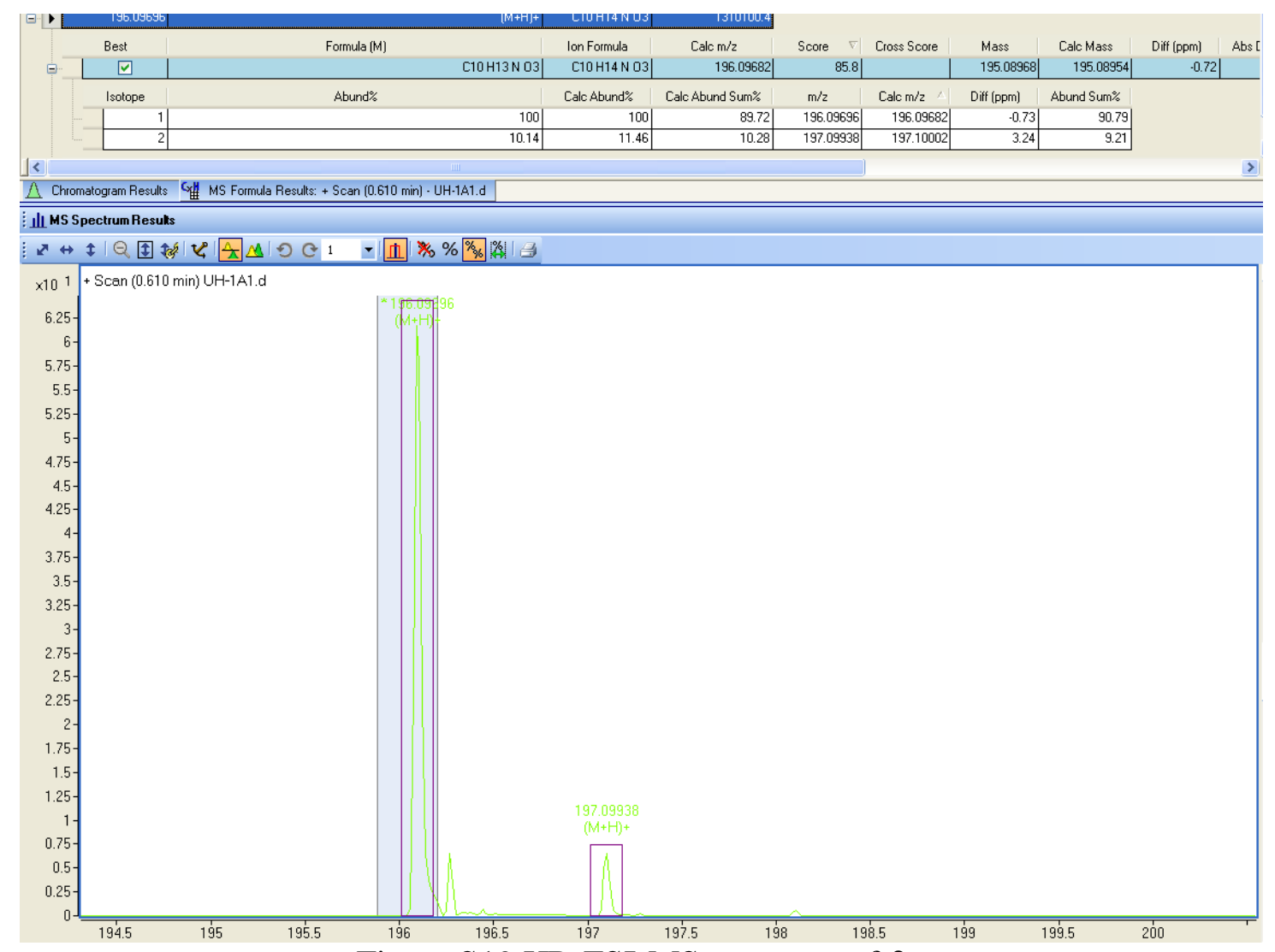

Figure S19.HR-ESI-MS spectrum of 2. 


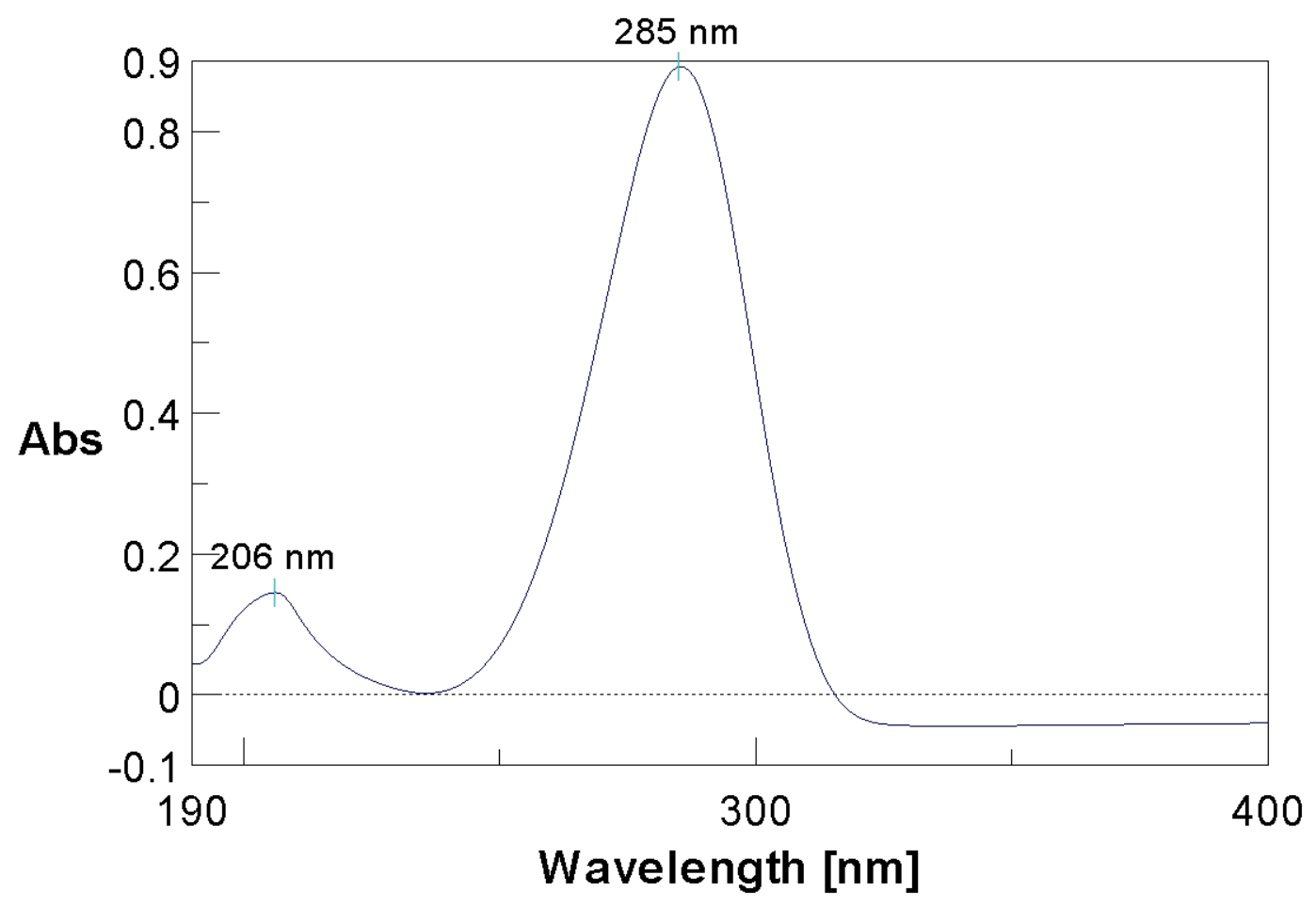

Figure S20. UV spectrum of 2 in $\mathrm{CH}_{3} \mathrm{OH}$.

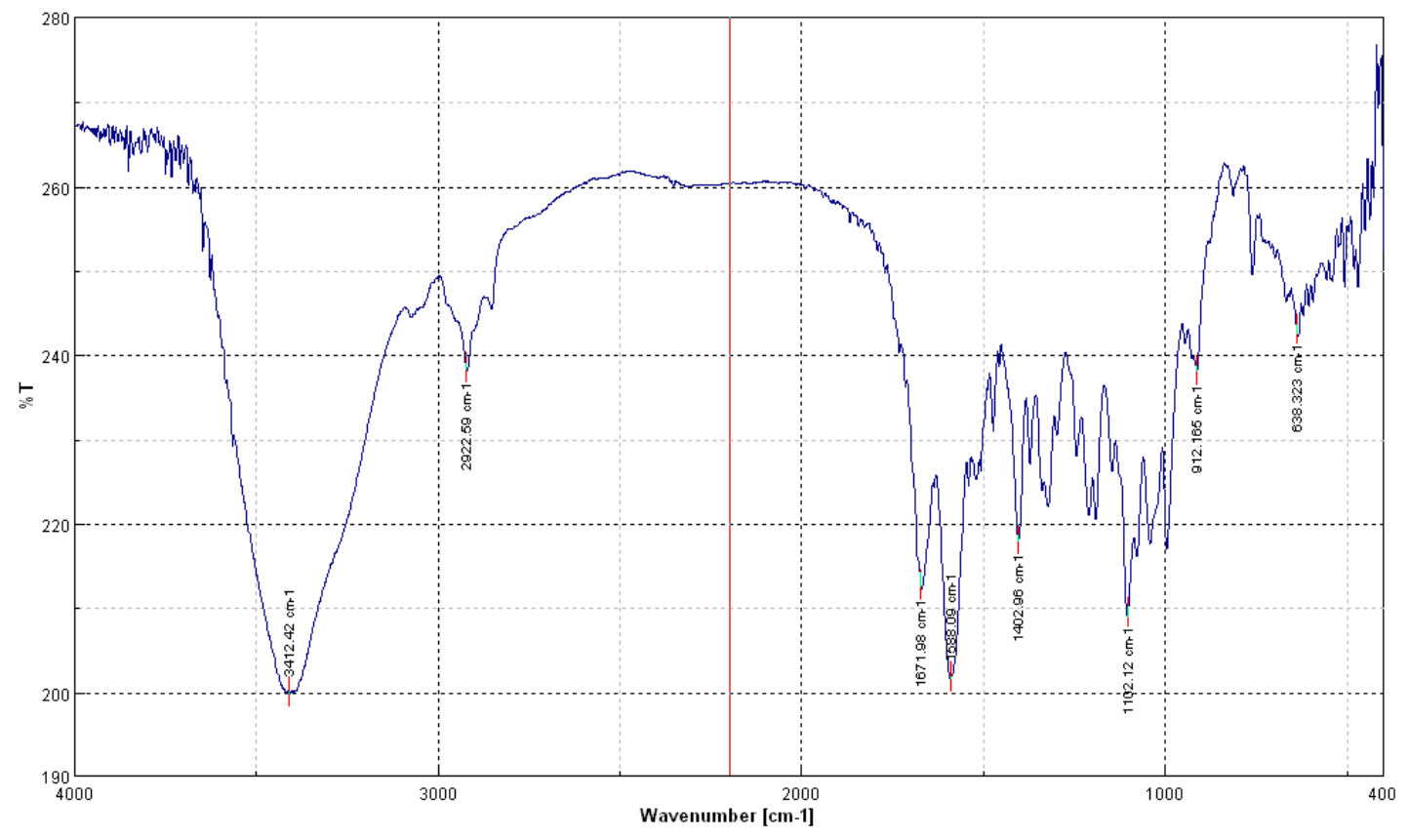

Figure S21. IR spectrum of 2 in $\mathrm{CH}_{3} \mathrm{OH}$. 


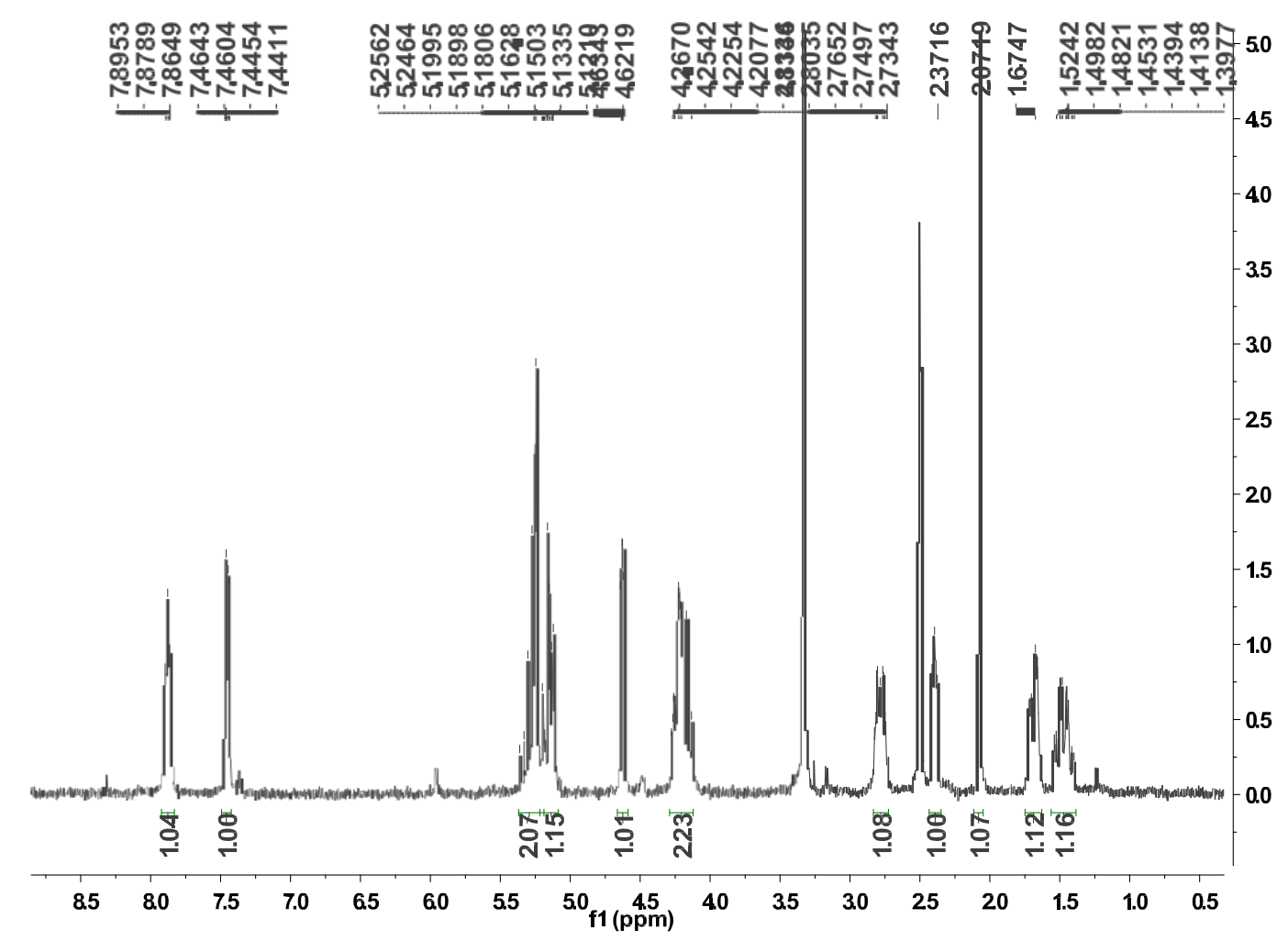

Figure S22. ${ }^{1} \mathrm{H}$ NMR spectrum of compound 3 in DMSO- $d_{6}(300 \mathrm{MHz})$.

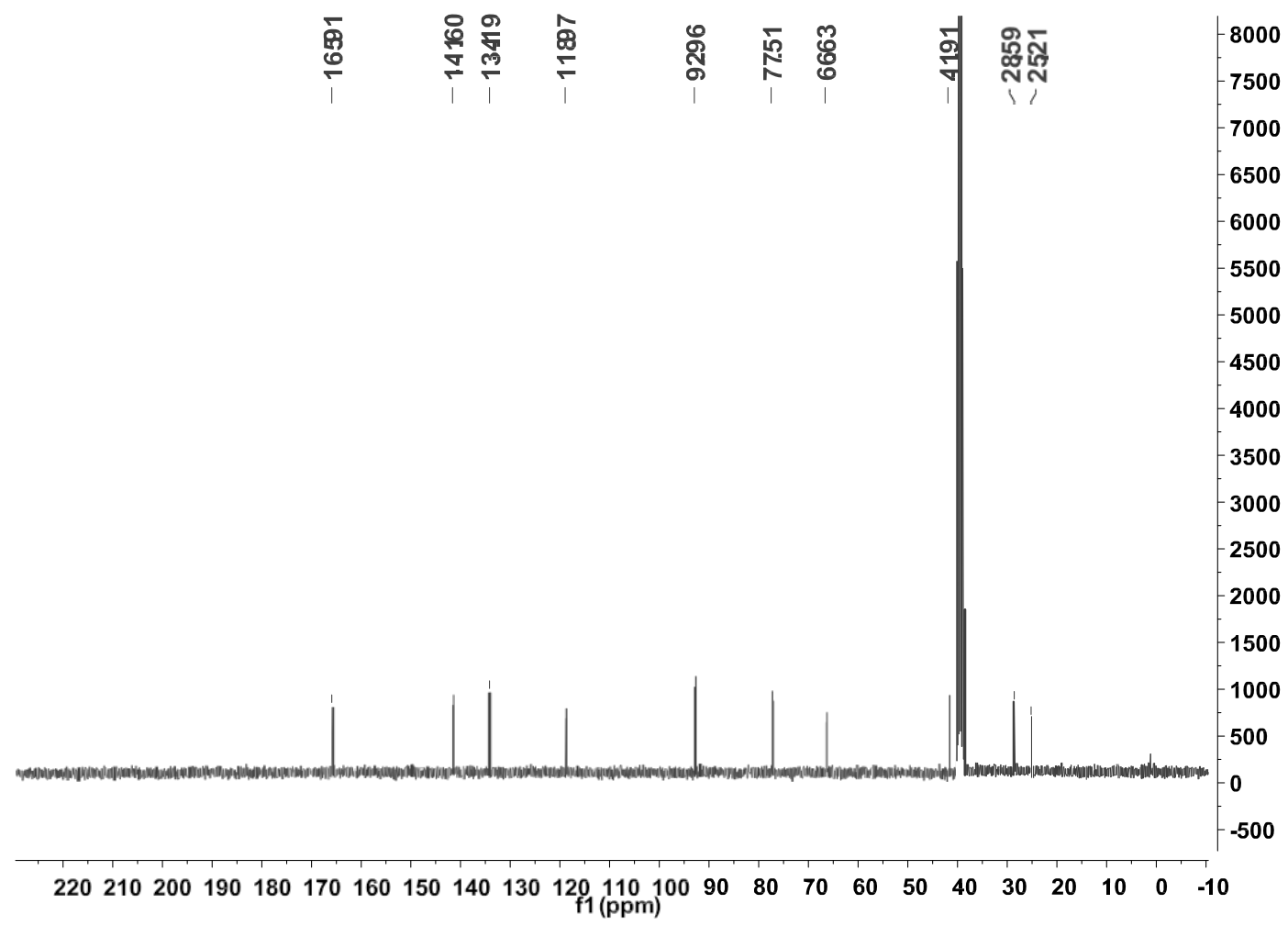

Figure S23. ${ }^{13} \mathrm{C}$ spectrum of compound 3 in DMSO-d $d_{6}(75 \mathrm{MHz})$. 


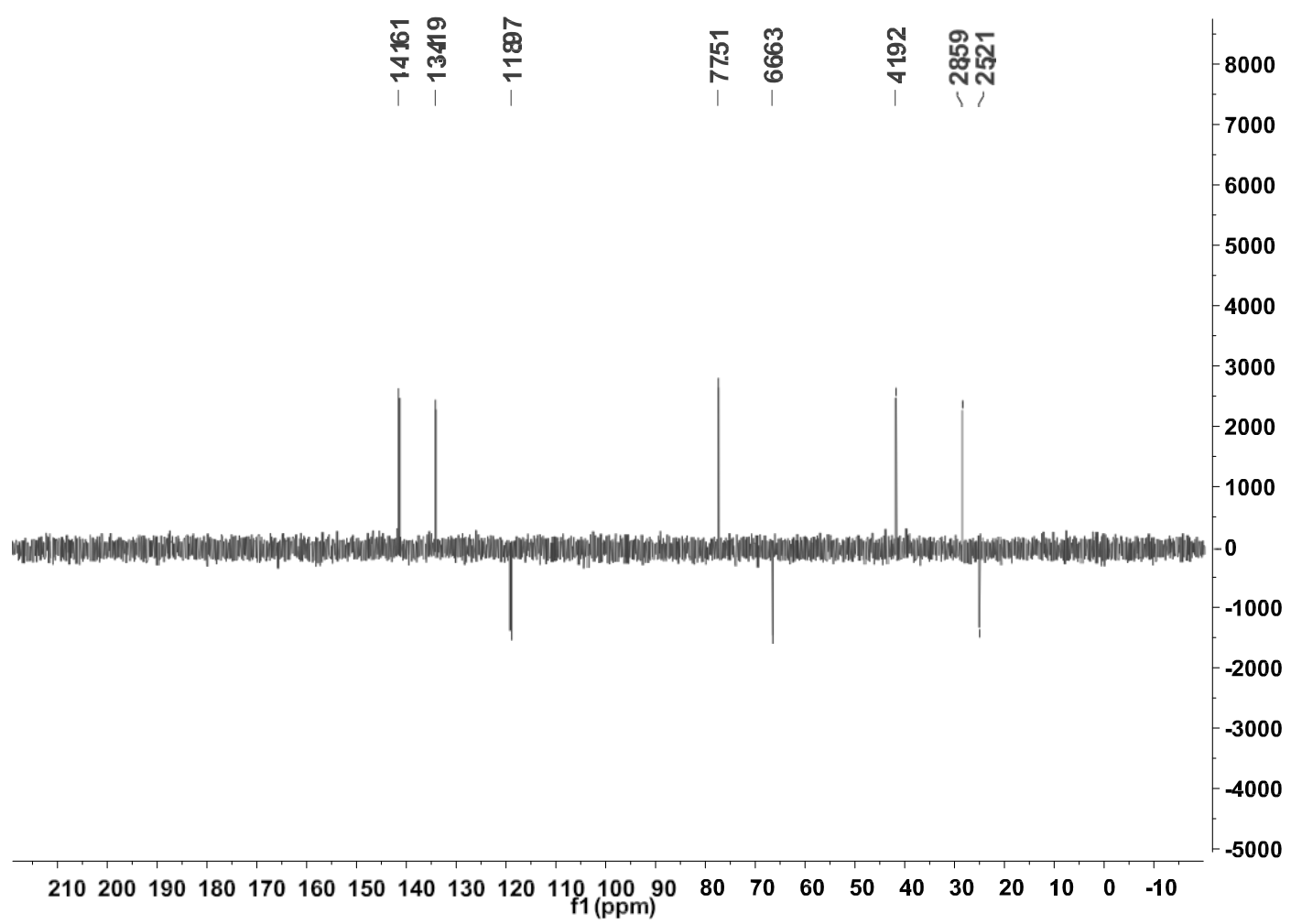

Figure S24. DEPT-135 spectrum of compound 3 in DMSO- $d_{6}(75 \mathrm{MHz})$.

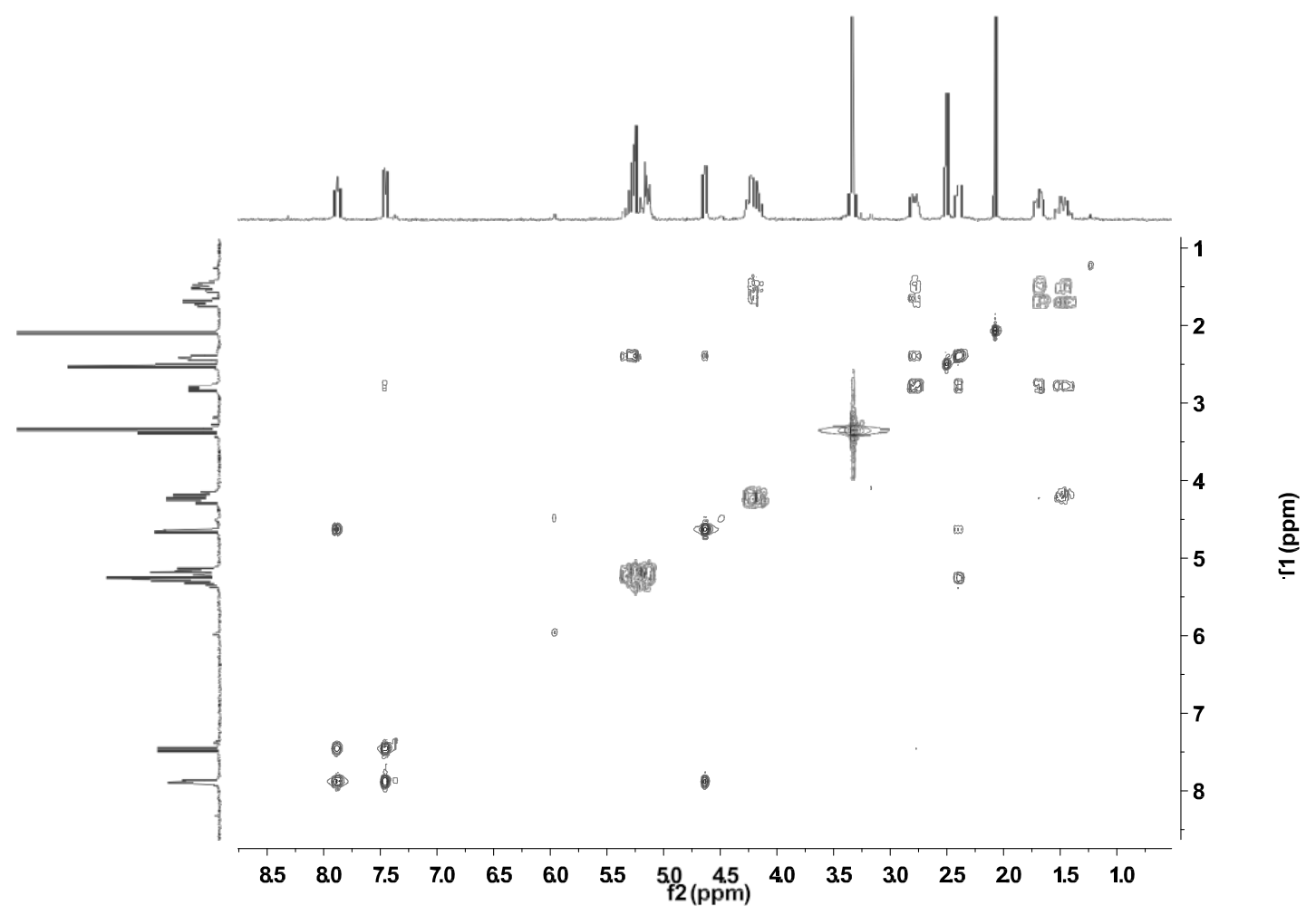

Figure S25. ${ }^{1} \mathrm{H}-{ }^{1} \mathrm{H}$ COSY spectrum of compound 3 in DMSO- $d_{6}$. 


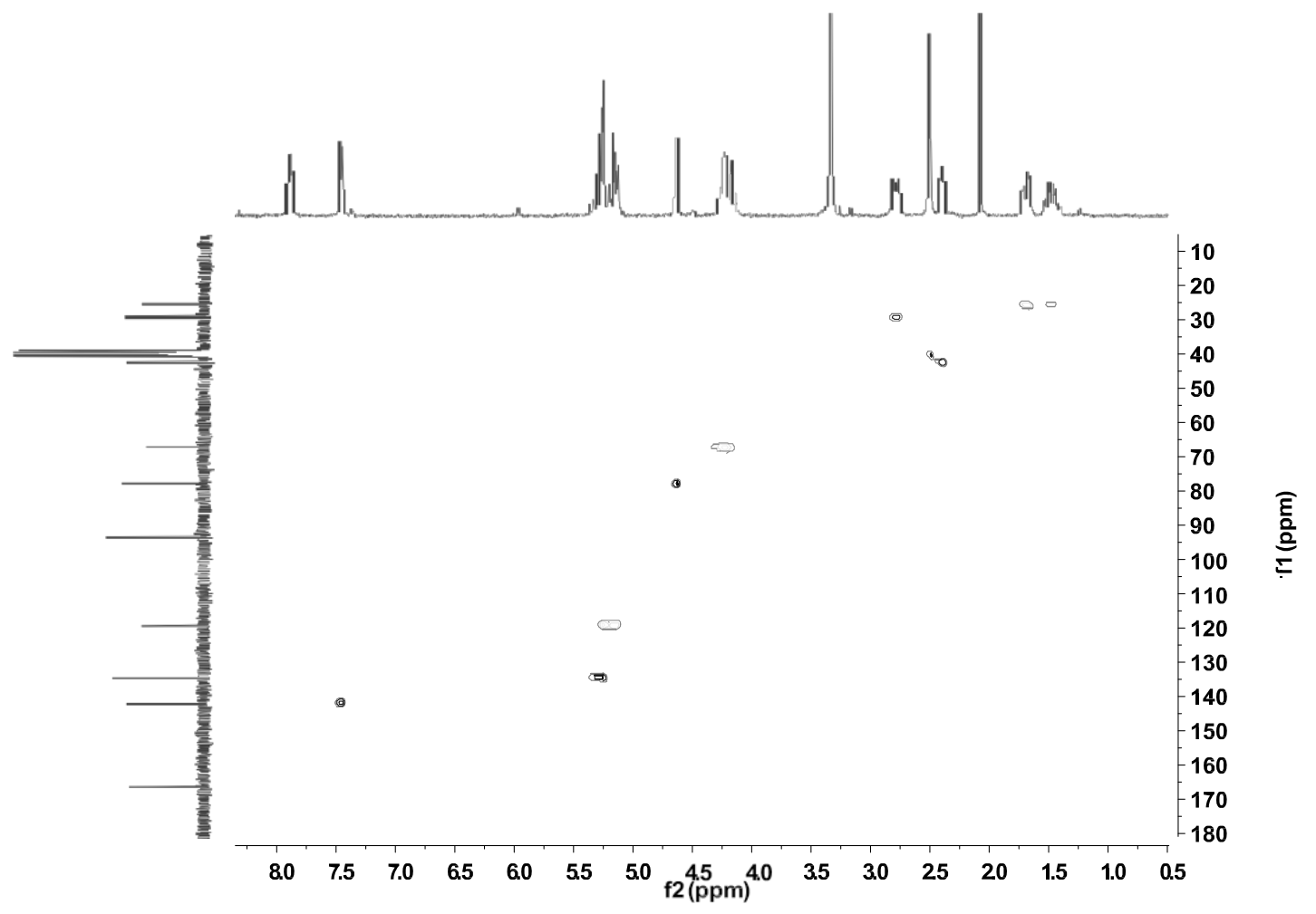

Figure S26. HSQC spectrum of compound 3 in DMSO- $d_{6}$.

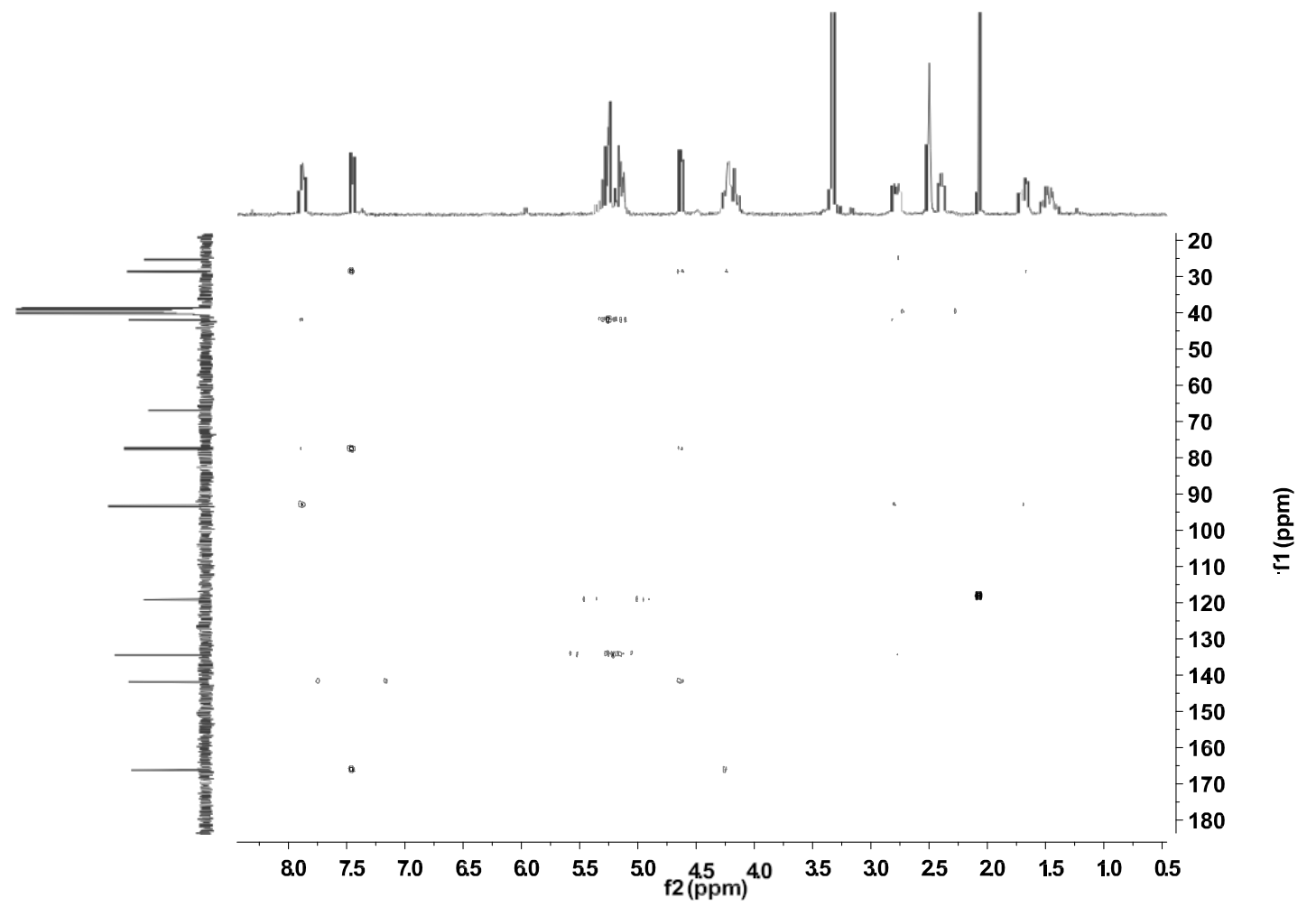

Figure S27. HMBC spectrum of compound 3 in DMSO- $d_{6}$. 


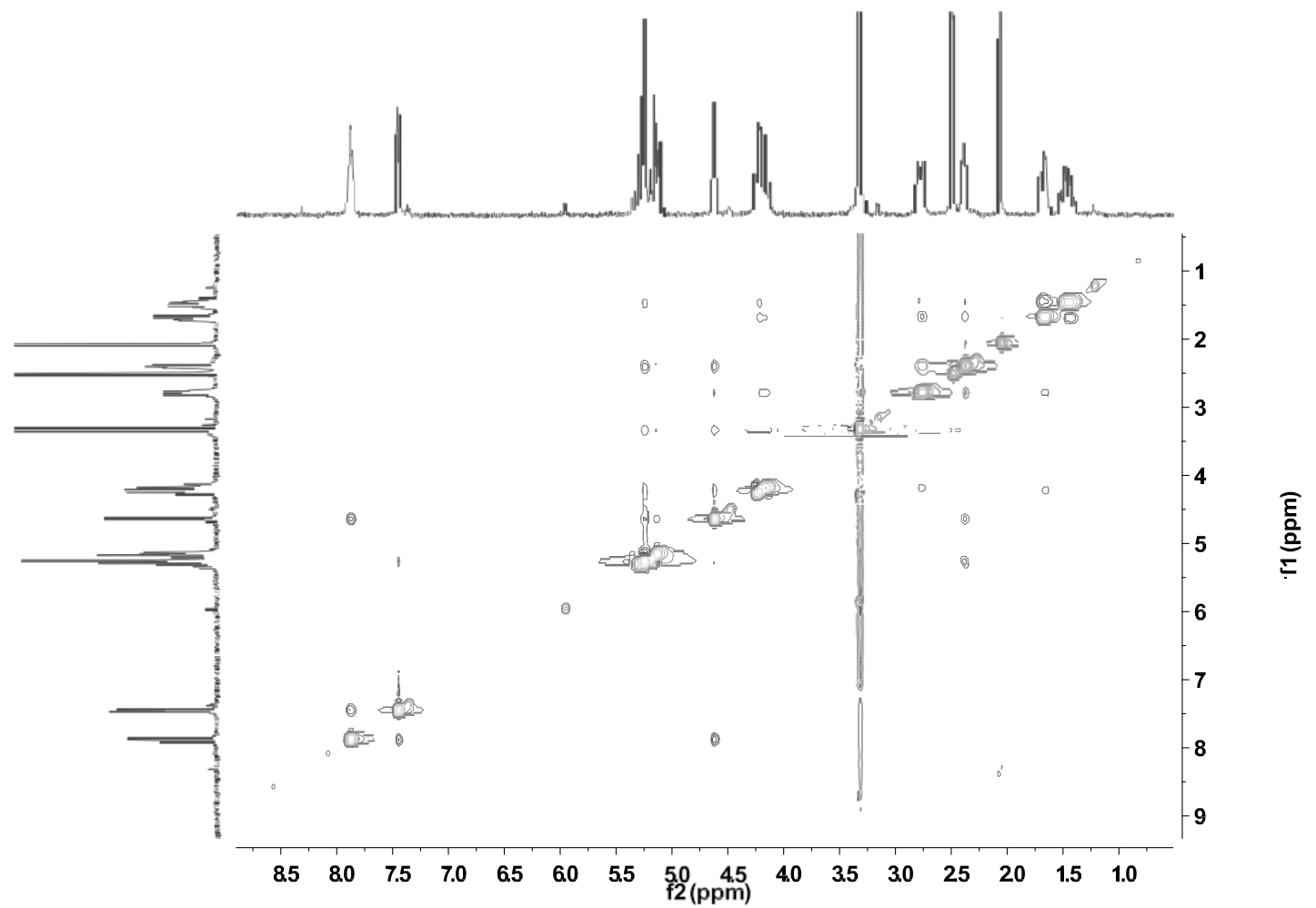

Figure S28. NOESY spectrum of compound 3 in DMSO- $d_{6}$. 


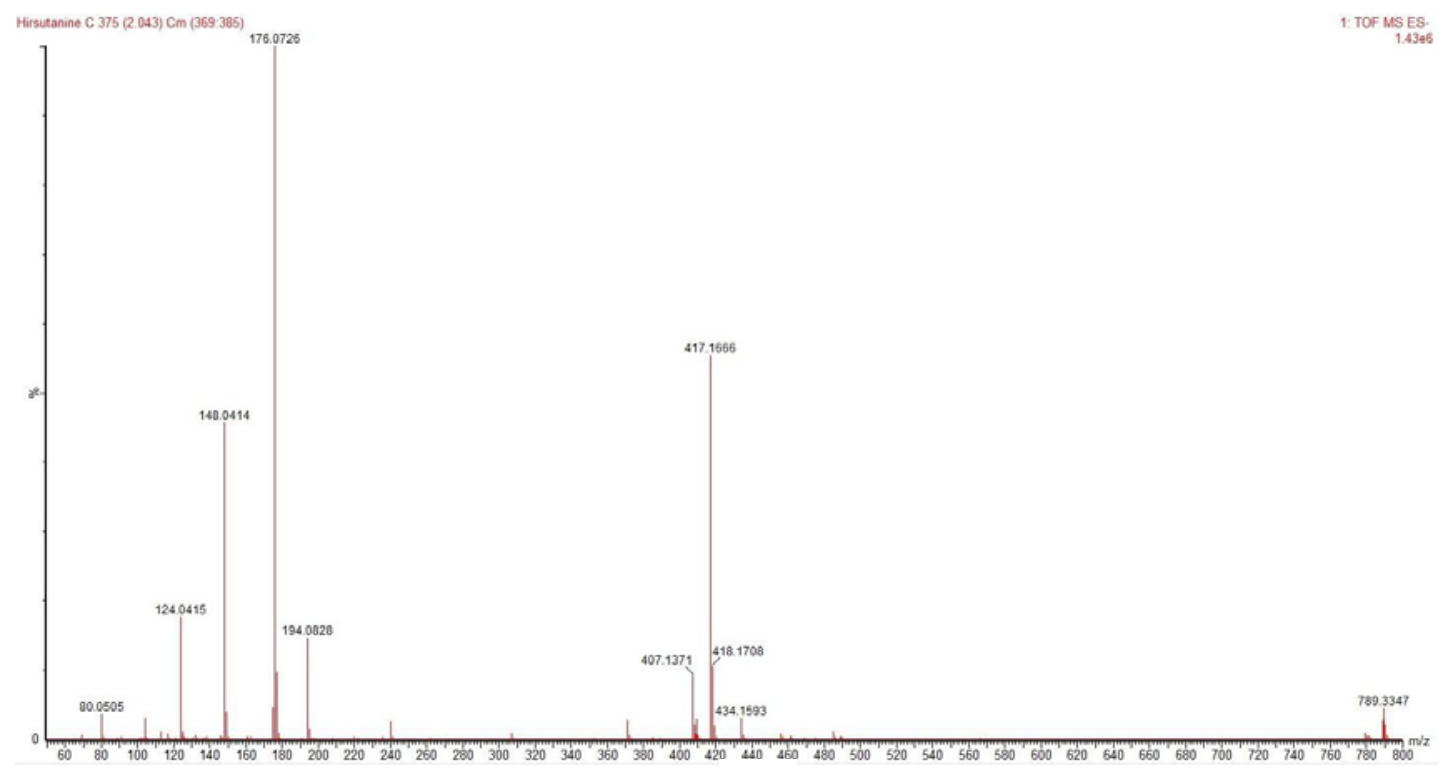

a
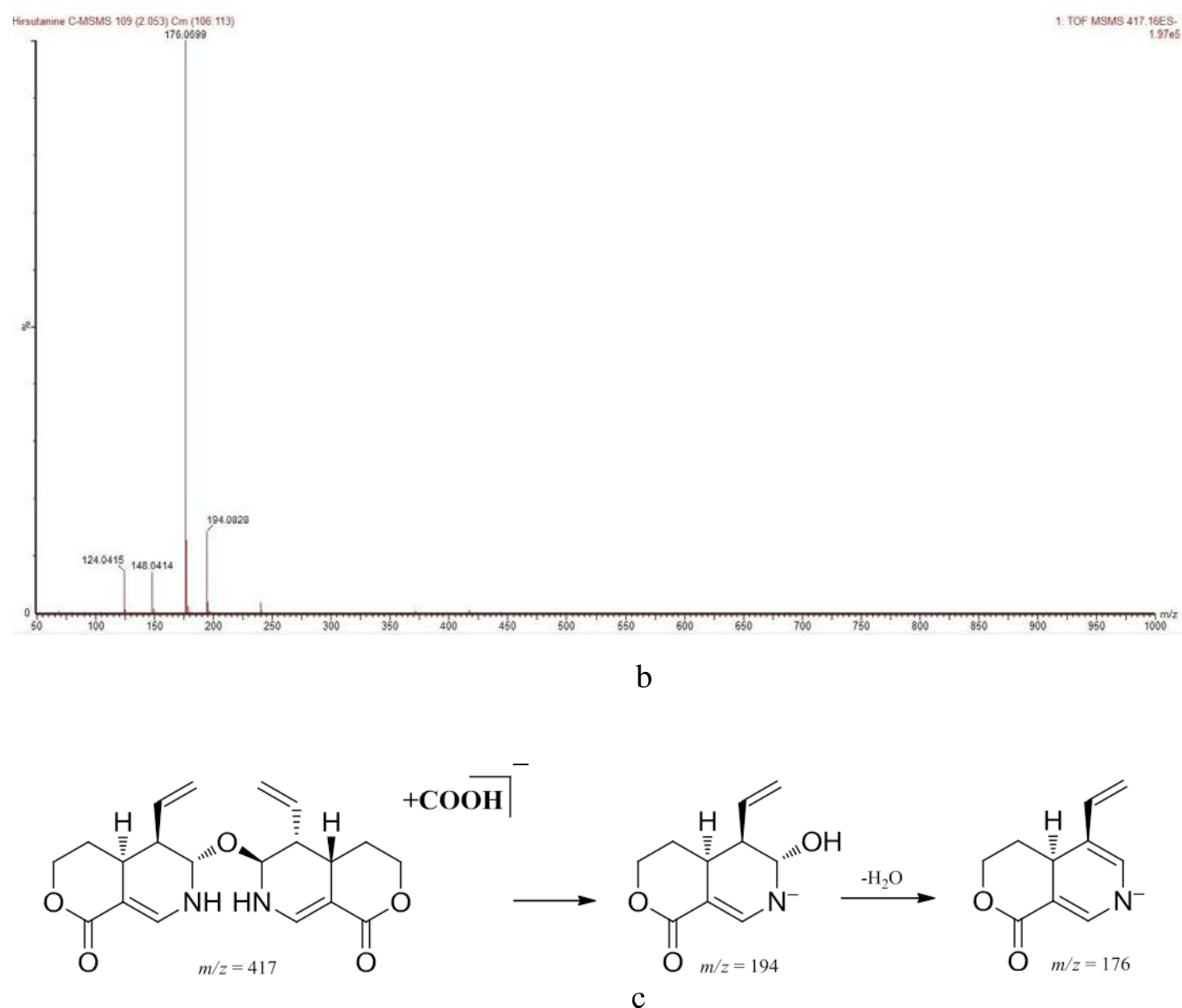

Figure S29. HR-ESI-MS (a) and MS/MS (b) of compound 3 (negative mode) and the proposed fragmentation pathway (c). 


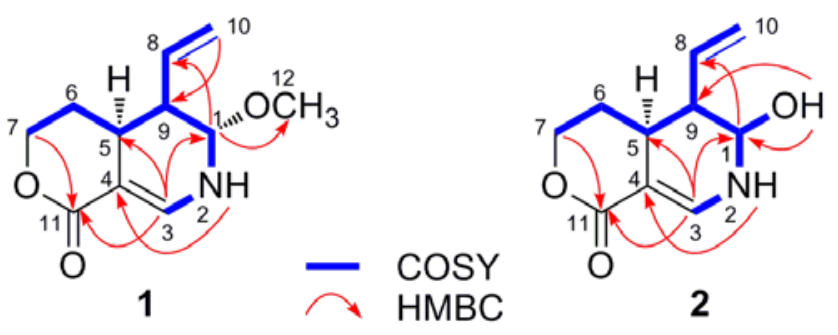

Figure S30. Key ${ }^{1} \mathrm{H}-{ }^{1} \mathrm{H}$ COSY and HMBC correlations of $\mathbf{1}$ and 2.

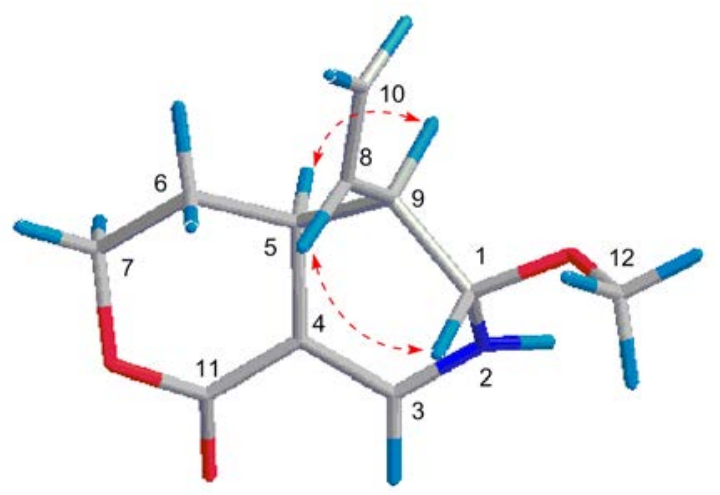

Figure S31. Key NOESY correlations of $\mathbf{1}$.

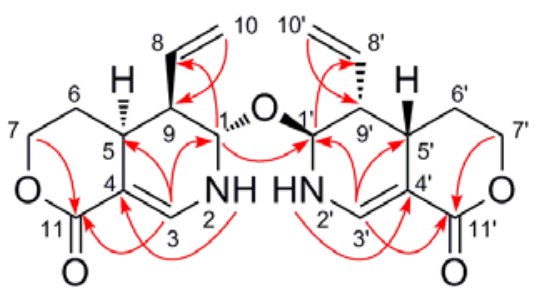

Figure S32. Key HMBC correlations of $\mathbf{3}$. 